SLAC-PUB-13183

arXiv:0803.3085v2[hep-th]

March 2008

\title{
Monodromy in the CMB: Gravity Waves and String Inflation
}

\author{
Eva Silverstein and Alexander Westphal \\ SLAC and Department of Physics, Stanford University, Stanford CA 94305
}

We present a simple mechanism for obtaining large-field inflation, and hence a gravitational wave signature, from string theory compactified on twisted tori. For Nil manifolds, we obtain a leading inflationary potential proportional to $\phi^{2 / 3}$ in terms of the canonically normalized field $\phi$, yielding predictions for the tilt of the power spectrum and the tensor-to-scalar ratio, $n_{s} \approx 0.98$ and $r \approx 0.04$ with 60 e-foldings of inflation; we note also the possibility of a variant with a candidate inflaton potential proportional to $\phi^{2 / 5}$. The basic mechanism involved in extending the field range - monodromy in D-branes as they move in circles on the manifold - arises in a more general class of compactifications, though our methods for controlling the corrections to the slow-roll parameters require additional symmetries. 


\section{Contents}

1 Introduction and Motivation $\quad 2$

2 Towards D4-brane Inflation on Twisted Tori 4

2.1 Compactification Manifold, Monodromies, and Geometric Field Range . . . . 4

2.2 Dynamics of wrapped D4-branes . . . . . . . . . . . . 7

2.3 The curvature-induced inflaton potential . . . . . . . . . . 8

2.3.1 Sol manifolds . . . . . . . . . . . . . . . . 12

2.3.2 Our main example: large $u_{1}$ and the $\phi^{2 / 3}$ potential . . . . . . . 12

2.3.3 A potential variant with $\tilde{V}(\tilde{\phi}) \propto \tilde{\phi}^{2 / 5} \ldots \ldots \ldots$. . . . . . . . . . . . . . .

2.4 Consistency with Moduli Stabilization and Background Geometry . . . . . 14

2.4.1 Condition $V_{\mathcal{R}}(\phi)<\mathcal{U}_{\text {mod, } \mathcal{R}} \ldots \ldots \ldots \ldots \ldots$. . . . . . . . . . . . . . . .

2.4.2 Moduli Shifts and the Inflaton potential . . . . . . . . . . . 16

2.4 .3 Back reaction of branes in $10 d \ldots \ldots \ldots$

2.5 Basic Observational Constraints: e-foldings and power spectrum . . . . . . 18

2.6 Conditions for Eternal inflation . . . . . . . . . . . . . . 19

2.7 Specific results in a concrete Nil manifold construction of dS vacua . . . . 20

2.7.1 Conditions for a Parametric Effect . . . . . . . . . . . 22

3 Theory Foregrounds: Systematic Analysis of Corrections 23

3.1 Contributions to $V, \epsilon$, and $\eta$ from Moduli-Stabilizing Ingredients . . . . . 24

3.1.1 A specific setup for string-theoretic $\phi^{2 / 3}$ inflation . . . . . . . . 26

3.1.2 A setup for the version with a $\tilde{\phi}^{2 / 5}$ potential . . . . . . . . . 28

3.1.3 General comments on the $\eta$ problem . . . . . . . . . . . 29 29

3.1.4 The Standard Model . . . . . . . . . . . . . . . 30

$3.2 \alpha^{\prime}$ and Loop Corrections . . . . . . . . . . . . . . . . 30

4 Observational Predictions $\quad 33$

5 Discussion 


\section{Introduction and Motivation}

It is of significant interest to develop inflationary mechanisms and models within string theory, as a UV complete theory of gravity. One reason for this is that inflationary models are UV sensitive: generic dimension six Planck-suppressed operators contribute order 1 corrections to the (generalized) slow roll parameters such as $\epsilon=-\frac{\dot{H}}{H^{2}}$. These must be at most of order $10^{-2}$ so as to obtain and sustain inflation - nearly constant $H=\dot{a} / a$ - for sufficiently long to solve the standard cosmological problems by increasing the scale $a(t)$ of the universe by a factor of $e^{60}[1]$. Other, related motivations for studying inflation in string theory include its role in discovering new mechanisms for inflation, and the potential for correlating (or anti-correlating) specific classes of field-theoretic inflationary models and signatures with classes of string compactifications 1

In this paper, we propose a new class of string inflation models arising in compactifications on manifolds with metric flux such as Nil manifolds which contain tori twisted over circles [3, 4]. A simple geometric feature of these spaces - monodromies in the one-cycles of the tori as one moves around the circle 2 - leads to large field ranges for the collective coordinates for wrapped D4-branes. This, combined with a detailed analysis of the effective action and the dynamical constraints on the mutual consistency of inflation and moduli stabilization, leads to a mechanism for large-field inflation with a $\phi^{2 / 3}$ potential for the canonically normalized inflaton field $\phi$ (a variant of one of the earliest field-theoretic proposals for inflation [5]). As in [5], we find that symmetries are very useful for helping to control the inflaton trajectory.

Because the suppression of the generalized slow-roll parameters $\epsilon$ and $\eta$ must hold for every point on the inflaton trajectory, it has a priori appeared particularly difficult to formulate large-field inflation, in which the canonically normalized inflaton field $\phi$ rolls over a distance in field space large compared to the four-dimensional Planck mass scale: $\Delta \phi \gg M_{\mathrm{P}}$. These large-field models are especially interesting from the observational point of view, because of the Lyth bound [6] showing that for any single-field model of inflation, observable tensor modes in the CMB require a super-Planckian field range. Upcoming CMB observations are projected to detect or constrain the tensor to scalar ratio $r$ at the level $r \gtrsim 0.01$ (see for example $[7,8]$ ), while existing and upcoming satellite experiments also significantly constrain the tilt of the spectrum [9].

There are two types of conditions which must be satisfied in order to obtain large-field inflation, which we will refer to as geometrical and dynamical criteria, respectively. First, the approximate moduli space of the scalar inflaton must extend over a distance greater than $M_{\mathrm{P}}$. Secondly the inflaton dynamics in this region $\Delta \phi \gg M_{\mathrm{P}}$ must be consistent with the stabilization of the underlying string compactification and with the smallness of the slow-

\footnotetext{
${ }^{1}$ For reviews of many works representing significant recent progress along these lines, see [2].

${ }^{2}$ This effect was discovered in a broad class of backgrounds - including non-geometric ones - in [10]. It would be very interesting to study our mechanism in this larger setting.
} 
roll parameters. In some interesting cases, even obtaining the possibility, geometrically, of a large field range proved impossible [11]. There are other interesting mechanisms which do not exhibit such a geometric limitation - such as [12, a theory of assisted inflation with multiple axions 3 and [15], brane inflation with multiple or wrapped branes rather than single D3-branes. In many of these cases, the dynamical self-consistency criteria happened to become important parametrically right at $\Delta \phi=M_{\mathrm{P}}$, leading to some speculation that a no-go theorem for gravity waves from string inflation might hold.

Controlling inflation in string theory is a somewhat laborious process, as it requires stabilizing the modulit. This is because one must satisfy the generalized slow roll conditions in every direction in field space in order to obtain inflation. Because moduli stabilization itself is somewhat complicated, and outlined so far only for special corners of the theory where control is most easily available, our knowledge of the space of possible top-down inflationary mechanisms and models is limited.

Recently a new class of de Sitter models in string theory was proposed in [4] in the context of ten-dimensional type IIA string theory compactified on Nil (or Sol) manifolds with orientifold 6-planes, fivebranes, and fluxes (complementing other work [17] showing that Calabi-Yau manifolds with a subset of allowed ingredients can be cleanly excluded). In this class of string compactifications, we find that inflation arises in a relatively straightforward way from the motion of a D4-brane wrapped on a one-cycle of the compactification - a brane which experiences a monodromy, not coming back to itself as it moves around a circle in the manifold. The resulting geometrically large field range yields a candidate large-field model, in which the brane executes multiple motions around the compact manifold during inflation. The corresponding inflaton potential descending from the D4-brane action turns out to be a fractional power, $V_{\mathcal{R}}(\phi) \sim \mu^{10 / 3} \phi^{2 / 3}$ for the appropriate regime in $\phi$ in the simplest case which we analyze in detail 5 As far as dynamical criteria go, this potential remains subdominant to the moduli potential over the requisite super-Planckian range of $\phi$, while a related $m^{2} \phi^{2}$ region of the potential is cleanly excluded on these grounds. We find further that the problem of suppressing the slow-roll parameters $\epsilon$ and $\eta \sim \ddot{\phi} /(H \dot{\phi})$ in this background is alleviated by its symmetries, and by the fact that the brane circles the same manifold multiple times during inflation. By considering moduli-fixing ingredients which are all extensive in the direction of the motion of the brane, we find that it is possible to avoid order 1 contributions to $\epsilon$ and $\eta$ which would otherwise be there, and on this basis we argue for the existence of models realizing our mechanism. Given that, the (fractional) power law potential yields predictions for the tilt $n_{s}-1$ of the spectrum of density perturbations, and

\footnotetext{
${ }^{3}$ Axions can play an important role in small-field string theoretic inflation as well; see [13] for an interesting recent example based partly on [14].

${ }^{4}$ For reviews of various aspects as well as references of this subject, see e.g. [16].

${ }^{5} \mathrm{~A}$ slight variant of this yields a candidate $\phi^{2 / 5}$ potential which we will also discuss; it would be interesting to analyze systematically the range of possibilities arising from branes with monodromies in more general backgrounds with curvature and flux.
} 
the tensor to scalar ratio $r$, which we review in our final section.

\section{Towards D4-brane Inflation on Twisted Tori}

\subsection{Compactification Manifold, Monodromies, and Geometric Field Range}

The simplest example of a Nil manifold suffices to exhibit our mechanism for extending the field range. A Nil 3-manifold is obtained by compactifying the nil geometry

$$
\begin{aligned}
d s_{N i l}^{2} & =L_{u_{1}}^{2} d u_{1}^{2}+L_{u_{2}}^{2} d u_{2}^{2}+L_{x}^{2}\left(d x+\frac{M}{2}\left[u_{1} d u_{2}-u_{2} d u_{1}\right]\right)^{2} \\
& =L_{u_{1}}^{2} d u_{1}^{2}+L_{u_{2}}^{2} d u_{2}^{2}+L_{x}^{2}\left(d x^{\prime}+M u_{1} d u_{2}\right)^{2}
\end{aligned}
$$

(where $x^{\prime}=x-\frac{M}{2} u_{1} u_{2}$ ) by a discrete subgroup of the isometry group

$$
\begin{aligned}
t_{x}:\left(x, u_{1}, u_{2}\right) & \rightarrow\left(x+1, u_{1}, u_{2}\right), \\
t_{u_{1}}:\left(x, u_{1}, u_{2}\right) & \rightarrow\left(x-\frac{M}{2} u_{2}, u_{1}+1, u_{2}\right), \\
t_{u_{2}}:\left(x, u_{1}, u_{2}\right) & \rightarrow\left(x+\frac{M}{2} u_{1}, u_{1}, u_{2}+1\right)
\end{aligned}
$$

This Nil 3-manifold $\mathcal{N}_{3}$ can be described as follows. For each $u_{1}$, there is a torus in the $u_{2}$ and $x^{\prime} \equiv x-\frac{M}{2} u_{1} u_{2}$ directions. Moving along the $u_{1}$ direction, the complex structure $\tau$ of this torus goes from $\tau \rightarrow \tau+M$ as $u_{1} \rightarrow u_{1}+1$. The projection by $t_{u_{1}}$ identifies these equivalent tori 6

At all values $u_{1}=j / M$ for integer $j$, the two-torus in the $u_{2}-x^{\prime}$ directions is equivalent to a rectangular torus

$$
d s_{r e c t}^{2} \equiv L_{x}^{2} d y_{1}^{2}+L_{u_{2}}^{2} d y_{2}^{2}, \quad\left(y_{1}, y_{2}\right) \equiv\left(y_{1}+n_{1}, y_{2}+n_{2}\right)
$$

(since $\tau \rightarrow \tau+1$ as $j \rightarrow j+1$ ). These coordinates $y_{1}$ and $y_{2}$ are related to $x^{\prime}$ and $u_{2}$ by an $S L(2, Z)$ transformation. The 1 -cycle traced out by $u_{2}=\lambda, \lambda \in(0,1)$ becomes a cycle $\left(y_{1}, y_{2}\right)=(M \lambda, \lambda)$ as $u_{1} \rightarrow u_{1}+1$. Applied to wrapped branes, this monodromy will yield a simple mechanism for obtaining a large field range, as follows.

Consider a D4-brane wrapped along the $u_{2}$ direction, and sitting at a point in the $u_{1}$ direction. (As we will discuss further below, we must also include a compensating source of $\overline{\mathrm{D}} 4$-brane charge.) This wrapped D4-brane contributes a potential energy in four dimensions of order $\frac{L_{4 B}}{(2 \pi)^{4}\left(\alpha^{\prime}\right)^{2} g_{s}}$, where $L_{4 B} \sqrt{\alpha^{\prime}}$ is the length of the cycle wrapped by the D4-brane, $\alpha^{\prime}$ is the inverse string tension and $g_{s}$ the ten-dimensional string coupling. At $u_{1}=0$, this

\footnotetext{
${ }^{6}$ The directions $u_{1}$ and $u_{2}$ are on the same footing; similar statements apply with the two interchanged and with $x^{\prime}$ replaced by $x^{\prime \prime} \equiv x+\frac{M}{2} u_{1} u_{2}$.
} 


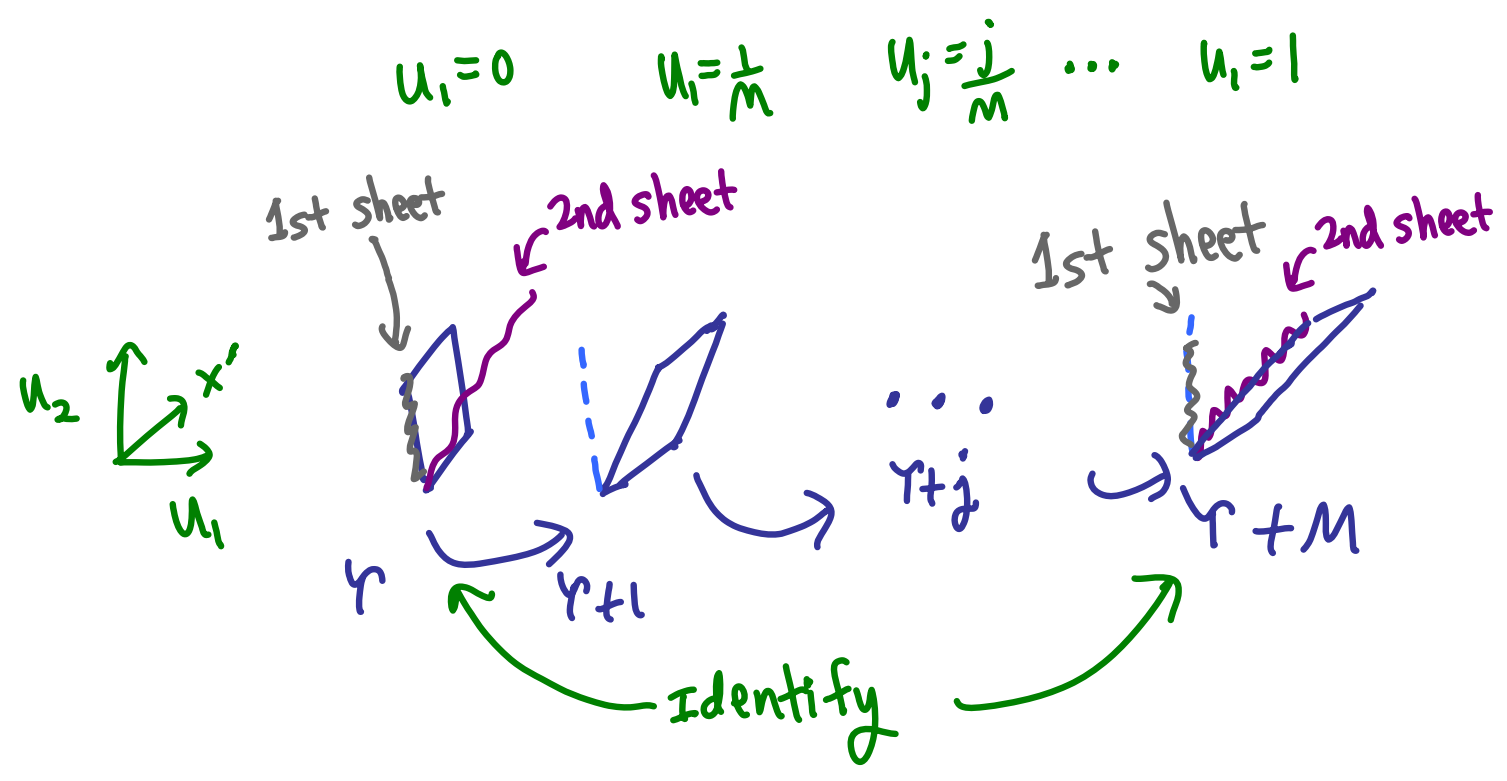

Figure 1: Monodromy of branes on a compact Nil 3-manifold. Start with a minimal-length D4-brane wrapped in the $u_{2}$ direction (indicated in grey). Moving it away from $u_{1}=0$ increases its mass since the cycle it wraps becomes longer. When it moves once around the compact $u_{1}$ direction, it has undergone a monodromy, now wrapping the cycle indicated in purple.

length is $L_{u_{2}} \sqrt{\alpha^{\prime}}$, and as a function of $u_{1}$ it is given by $L_{4 B}=\sqrt{L_{u_{2}}^{2}+M^{2} L_{x}^{2} u_{1}^{2}}$. That is, as the D4-brane moves in the $u_{1}$ direction away from $u_{1}=0$, it becomes heavier. We will shortly derive the form of this curvature-induced potential as a function of the canonically normalized field $\phi$ related to $u_{1}$.

We can now immediately see that the range of the field is not geometrically limited in the way it was for a single D3-brane in a Calabi-Yau manifold [11. In the case considered in [11], the approximate moduli space of the brane and the four-dimensional Planck mass are both determined by the volume of the compactification. In the present case, the fourdimensional Planck mass $M_{\mathrm{P}}$ is fixed as usual by the volume of the compactification (in the absence of large species enhancements to it [12], which we will exclude in assessing the corrections below). But the brane's field space is not constrained by the compactification volume, as a result of the monodromies just described. Consider the motion of the D4-brane in the $u_{1}$ direction. As the $u_{1}$ position of the $\mathrm{D} 4$-brane reaches 1 , the D4-brane has moved once around the compact manifold. However, it now lies on the cycle $\left(y_{1}, y_{2}\right)=(M \lambda, \lambda)$ in the two-torus (note that it still carries the same charge since the 1-cycle it wraps lies in the same homology class). The approximate moduli space of the brane lies on a subspace of the covering space of $\mathcal{N}_{3}$ - the space obtained by undoing the projection by $t_{u_{1}}$ in (2.2). Hence, at fixed four-dimensional Planck mass $M_{\mathrm{P}}$, the geometrical moduli space of a single wrapped D4-brane is unlimited in field range. 
We will seek a concrete model realizing this mechanism, on a product of two Nil 3manifolds with a D4-brane wrapped on a linear combination of their $u_{2}$ directions, whose motion in a linear combination of their $u_{1}$ directions describes inflation. (We will also consider a variant with motion along a linear combination of $u_{1}$ and $u_{2}$ directions.) Starting out away from the minimum of the candidate inflaton potential corresponds to beginning with a D4-brane wound up in the manner just described; as it unwinds by circling the $u_{1}$ directions multiple times it approaches the minimum of its potential and eventually exits from inflation.

So far there are some similarities between our mechanism for a geometrically large field range and previously proposed mechanisms such as [12, 15]. The fact that after moving once around the base circle in the $u_{1}$ direction our brane lies on the $(M, 1)$-cycle of the 2 -torus described above means that there is some sense in which it constitutes multiple or wrapped branes. As noted in the previous works, a large field range is not in itself sufficient to produce a consistent model of large-field inflation. We must check that the potential energy carried by the inflaton not back react significantly on the geometry and destabilize the compactification, and that all $\alpha^{\prime}$ and quantum corrections to the inflaton potential are small or can be tuned to preserve the smallness of the slow roll parameters everywhere on the inflaton trajectory.

In the previous examples, dynamical criteria of this sort happened to become important parametrically right at a field range $\Delta \phi$ of order $M_{\mathrm{P}}$. In our case, we will find that enforcing these dynamical criteria for control remains consistent with $\Delta \phi \gg M_{\mathrm{P}}$ in our Nil manifold compactification. We will be led to a window with fixed, small couplings and curvatures where our candidate model appears viable from the theoretical and observational points of view.

Clearly this monodromy effect - the fact that the D-brane approximate moduli space lives on a cover of the compactification - arises much more generally [10] than in the setup based on Nil 3-manifolds which we consider here. In particular, there are similar monodromies for Sol 3-manifolds, where the $S L(2, Z)$ transformation obtained in moving around the base circle is more general than $\tau \rightarrow \tau+M$. However, as we will see, in that case the dynamical consistency criteria - specifically the condition that the inflaton potential not exceed the moduli-stabilizing curvature potential energy - cleanly excludes this possibility .7 It would be interesting to perform a systematic study of models making use of this mechanism in more general compactifications such as [10, 18]. We turn next to a systematic analysis of the D4-brane dynamics in our Nil manifold case.

\footnotetext{
${ }^{7}$ However, there exist stabilization mechanisms with larger potential energy - such as those coming from hyperbolic compactifications and/or supercritical ones - as reviewed in [16], and these may accommodate a wider class of inflationary models.
} 


\subsection{Dynamics of wrapped D4-branes}

The effective action for the collective coordinates $X$ of our D4-brane in a given background, with dilaton $\Phi(X)$, metric $G_{M N}(X)$, Neveu-Schwarz potential $B_{M N}(X)$, and RR potentials $C^{(p)}(X)$ is

$$
\mathcal{S}_{\mathrm{D} 4}=-\int \frac{d^{5} \xi}{(2 \pi)^{4}\left(\alpha^{\prime}\right)^{5 / 2}} e^{-\Phi} \sqrt{\operatorname{det}\left(G_{M N}+B_{M N}\right) \partial_{\alpha} X^{M} \partial_{\beta} X^{N}}+\mathcal{S}_{C S}+\text { loops }
$$

This DBI action is self-consistently valid near solutions in which higher derivatives of the fields are negligible, and we will estimate the size of corrections to it in later sections. It is crucial in analyzing (2.4) to plug in the correct solutions for $G_{M N}, \Phi$, and $B_{M N}$, including all effects of the compactification [20, 21, 22, 23] and all relevant $\alpha^{\prime}$ and loop effects. In particular, the compactification affects the Green's functions determining fields emanating from sources inside it [20, 21, 22, 23] in a way that generically produces order 1 contributions to the slow-roll parameters in brane inflation models.

The Chern-Simons action is given by

$$
\mathcal{S}_{C S}=\frac{1}{(2 \pi)^{4}\left(\alpha^{\prime}\right)^{5 / 2}} \int\left(\sum_{p} C^{(p)}\right) e^{-B} e^{2 \pi \alpha^{\prime} F}
$$

where $F$ is the worldvolume gauge flux (which will play no role in our considerations). $\mathcal{S}_{C S}$ simplifies greatly in our situation, as follows. The background form fields in [4] preserve the Lorentz symmetry in four dimensions. Therefore (2.5) can only get contributions from $C_{0123 u_{2}}^{(5)}$, with legs along the five worldvolume dimensions of the D4-brane. In the original moduli-stabilized compactification, there is no such background field - the solutions in [4] did not make use of an $F_{6}$ flux in these directions (or its dual internal $F_{4}$ flux). However as mentioned above, we must have a source of $\overline{\mathrm{D} 4}$-brane charge in the compactification in order to satisfy Gauss' law for $C^{(5)}$. We will include this source in the form of flux below in \$3, arguing that its homogeneity suppresses its contributions to the slow-roll parameters in our model.

The background configurations of $\Phi, G_{M N}$, and $B_{M N}$ appearing in (2.4) are given by the nil geometry (2.1) plus contributions from the other ingredients required to stabilize the moduli (which in the case of the construction [4] consist of fivebranes, O6-planes, zero-form and six-form fluxes $m_{0}$ and $F_{6}$, and discrete Wilson lines built from the $B$ field) plus more general $\alpha^{\prime}$ and loop corrections. We will start by analyzing the contributions to the D4brane dynamics coming from the Nil geometry itself, deriving a curvature-induced potential $V_{\mathcal{R}}(\phi)$ for the canonically normalized field $\phi$ corresponding to the D4-brane's motion in the $u_{1}$ direction. A crucial requirement we must impose is that this candidate inflaton potential $V_{\mathcal{R}}(\phi)$ not destabilize the moduli or back react strongly on the geometry (or vice versa), and we find that this condition is straightforward to satisfy for a large range of the field $\phi$. In 
the relevant range, we find that the potential $V_{\mathcal{R}}(\phi)$ is well approximated by a power law potential $V(\phi) \propto \phi^{2 / 3}$, leading to specific predictions for two basic CMBR observables, $r$ (the tensor to scalar ratio) and $n_{s}$ (the tilt of the spectrum) which lie in an observationally accessible regime, near the central value for these quantities according to existing data.

We will then address in $\$ 3$ the other contributions from the remaining ingredients in the moduli stabilization mechanism [4], and from $\alpha^{\prime}$ and loop corrections to the background, arguing that for appropriate configurations of ingredients, these need not destabilize the resulting inflaton trajectory.

\subsection{The curvature-induced inflaton potential}

We consider type IIA string theory compactified to $4 \mathrm{~d}$ on an orientifold of a product of two identical Nil manifolds $\mathcal{N}_{3} \times \tilde{\mathcal{N}}_{3}$ [4]. The Nil 3-manifold $\mathcal{N}_{3}$ was described above, with metric

$$
\frac{d s^{2}}{\alpha^{\prime}}=L_{u_{1}}^{2} d u_{1}^{2}+L_{u_{2}}^{2} d u_{2}^{2}+L_{x}^{2}\left(d x^{\prime}+M u_{1} d u_{2}\right)^{2}
$$

and we have an isomorphic metric for the second Nil 3-manifold factor, with corresponding coordinates $\tilde{u}_{1}, \tilde{u}_{2}, \tilde{x}^{\prime}$.

Assume now a D4-brane wrapped along the $u_{2}$-direction (or a combination of the $u_{2}$ and

$\tilde{u}_{2}$ directions) and watch it as it moves along $u_{1}$ (or similarly a combination of the $u_{1}$ and $\tilde{u}_{1}$ directions). As explained above, the energy density of the D4-brane grows monotonically with increasing $u_{1}$ even as the brane moves along $u_{1}$ multiple times through the fundamental domain of the geometry. In order to analyze the resulting candidate inflaton dynamics, it is most convenient to transform to the canonically normalized scalar field $\phi$ corresponding to the motion of the D4-brane in the $u_{1}$ direction.

We start with the DBI action (2.4) for a single D4-brane, with zero $B_{(2)}$ in the worldvolume directions, and also vanishing worldvolume gauge field strength $F_{(2)}$. The fourdimensional Planck mass $M_{\mathrm{P}}$ is related to the string tension $1 / \alpha^{\prime}$ via

$$
\frac{1}{\alpha^{\prime}}=\frac{(2 \pi)^{7}}{2} g^{2} M_{\mathrm{P}}^{2} \quad \text { with }: \quad g^{2}=\frac{g_{s}^{2}}{L^{6} / 2}
$$

the 4-dimensional string coupling defined from the reducing the type IIA supergravity action $S_{\text {IIA }}=-1 / 2 \kappa^{2} \int d^{10} x \sqrt{-g} e^{-2 \phi} R+\ldots$ on the orientifold of $\mathcal{N}_{3} \times \tilde{\mathcal{N}}_{3}$ with volume $\mathcal{V}=L^{6} / 2$

The 'radial' modulus $L$ is defined in terms of the size moduli for the $u_{i}, \tilde{u}_{i}, x, \tilde{x}$ directions as $L^{3}=L_{u_{1}} L_{u_{2}} L_{x}=L_{\tilde{u}_{1}} L_{\tilde{u}_{2}} L_{\tilde{x}}$. As $u_{1}$ and $u_{2}$ are interchangeable in their respective roles before the introduction of the D4-brane, we define a common scale $L_{u}$ such that $L^{3}=L_{u}^{2} L_{x}$ and parametrize the anisotropy of the Nil manifold through the modulus

$$
\beta \equiv \frac{L_{u_{2}}}{L_{u_{1}}}=\frac{L_{u}^{2}}{L_{u_{1}}^{2}}=\frac{L_{u_{2}}^{2}}{L_{u}^{2}}
$$


which can be tuned by an appropriate choice of flux and brane quanta [4], as we will discuss further below. (Ultimately a 1 percent fine-tune will be needed.) In terms of these quantities, we obtain the following action for the fluctuations of the brane position $u_{1}$

$$
\begin{aligned}
S_{\mathrm{D} 4}\left[u_{1}\right] & =-\frac{1}{(2 \pi)^{4} g_{s} \alpha^{\prime 5 / 2}} \int_{\mathcal{M}_{4} \times u_{2}} \mathrm{~d}^{5} \xi \sqrt{-g_{4} g_{u_{2} u_{2}}\left(1-\alpha^{\prime} g_{u_{1} u_{1}} \dot{u}_{1}^{2}\right)} \\
& =-\frac{1}{(2 \pi)^{4} g_{s} \alpha^{\prime 2}} \int_{0}^{1} d u_{2} \int_{\mathcal{M}_{4}} \mathrm{~d}^{4} \xi \sqrt{-g_{4}} \sqrt{\left(\beta L_{u}^{2}+L_{x}^{2} M^{2} u_{1}^{2}\right)\left(1-\alpha^{\prime} \frac{L_{u}^{2}}{\beta} \dot{u}_{1}^{2}\right)} \\
& =-\frac{1}{(2 \pi)^{4} g_{s} \alpha^{\prime 2}} \int d^{4} x \sqrt{-g_{4}} \sqrt{\left(\beta L_{u}^{2}+L_{x}^{2} M^{2} u_{1}^{2}\right)\left(1-\alpha^{\prime} \frac{L_{u}^{2}}{\beta} \dot{u}_{1}^{2}\right)} .
\end{aligned}
$$

Upon expanding this action up to two derivatives we arrive at

$$
S_{\mathrm{D} 4}=\frac{1}{(2 \pi)^{4} g_{s} \alpha^{\prime 2}} \int d^{4} x \sqrt{-g_{4}}\left(\beta^{-1} L_{u}^{2} \sqrt{\beta L_{u}^{2}+L_{x}^{2} M^{2} u_{1}^{2}} \alpha^{\prime} \frac{\dot{u}_{1}^{2}}{2}-\sqrt{\beta L_{u}^{2}+L_{x}^{2} M^{2} u_{1}^{2}}+\ldots\right) .
$$

This action describes the dynamics of a non-canonically normalized scalar field with a positive definite potential. To exhibit the dynamics of $u_{1}$ we pass to a canonically normalized scalar field $\phi$ with kinetic term $S=\int d^{4} x \sqrt{-g_{4}} \dot{\phi}^{2} / 2$. In terms of the D4-brane position $u_{1}$ we then have

$$
\dot{\phi}=\phi^{\prime}\left(u_{1}\right) \dot{u}_{1} \quad \Leftrightarrow \quad \frac{\dot{\phi}^{2}}{2}=\phi^{\prime 2} \frac{\dot{u}_{1}^{2}}{2}
$$

and thus

$$
\begin{aligned}
& \phi^{\prime}\left(u_{1}\right)=\frac{L_{u}^{3 / 2} \beta^{-1 / 4}}{(2 \pi)^{2} \sqrt{g_{s} \alpha^{\prime}}}\left(1+\frac{M^{2} L_{x}^{2}}{\beta L_{u}^{2}} u_{1}^{2}\right)^{1 / 4} \\
& \Rightarrow \phi=\frac{L_{u}^{3 / 2} \beta^{-1 / 4}}{3(2 \pi)^{2} \sqrt{g_{s} \alpha^{\prime}}} u_{1}\left[F_{1, \frac{1}{2}, \frac{3}{4}, \frac{3}{2}}^{2}\left(-\frac{M^{2} L_{x}^{2}}{\beta L_{u}^{2}} u_{1}^{2}\right)+2\left(1+\frac{M^{2} L_{x}^{2}}{\beta L_{u}^{2}} u_{1}^{2}\right)^{1 / 4}\right]
\end{aligned}
$$

where $F_{p, q, r, s}^{2}(x)$ denotes a hypergeometric function. In terms of this canonically normalized scalar field $\phi$ the D4-brane action to quadratic order in the derivatives then reads

$$
S_{\mathrm{D} 4}=\int d^{4} x \sqrt{-g_{4}}\left[\frac{1}{2} \dot{\phi}^{2}-V_{\mathcal{R}}(\phi)\right]
$$

with

$$
V_{\mathcal{R}}(\phi)=\frac{\sqrt{\beta} L_{u}}{(2 \pi)^{4} g_{s} \alpha^{\prime 2}} \sqrt{1+\frac{M^{2} L_{x}^{2}}{\beta L_{u}^{2}} u_{1}^{2}(\phi)}
$$

where $u_{1}(\phi)$ is to be read as the inverse function of eq. (2.12). This inversion we could not do analytically. However, it is possible to invert $\phi\left(u_{1}\right)$ numerically which results in $V_{\mathcal{R}}(\phi)$ as displayed in Fig. 2. 


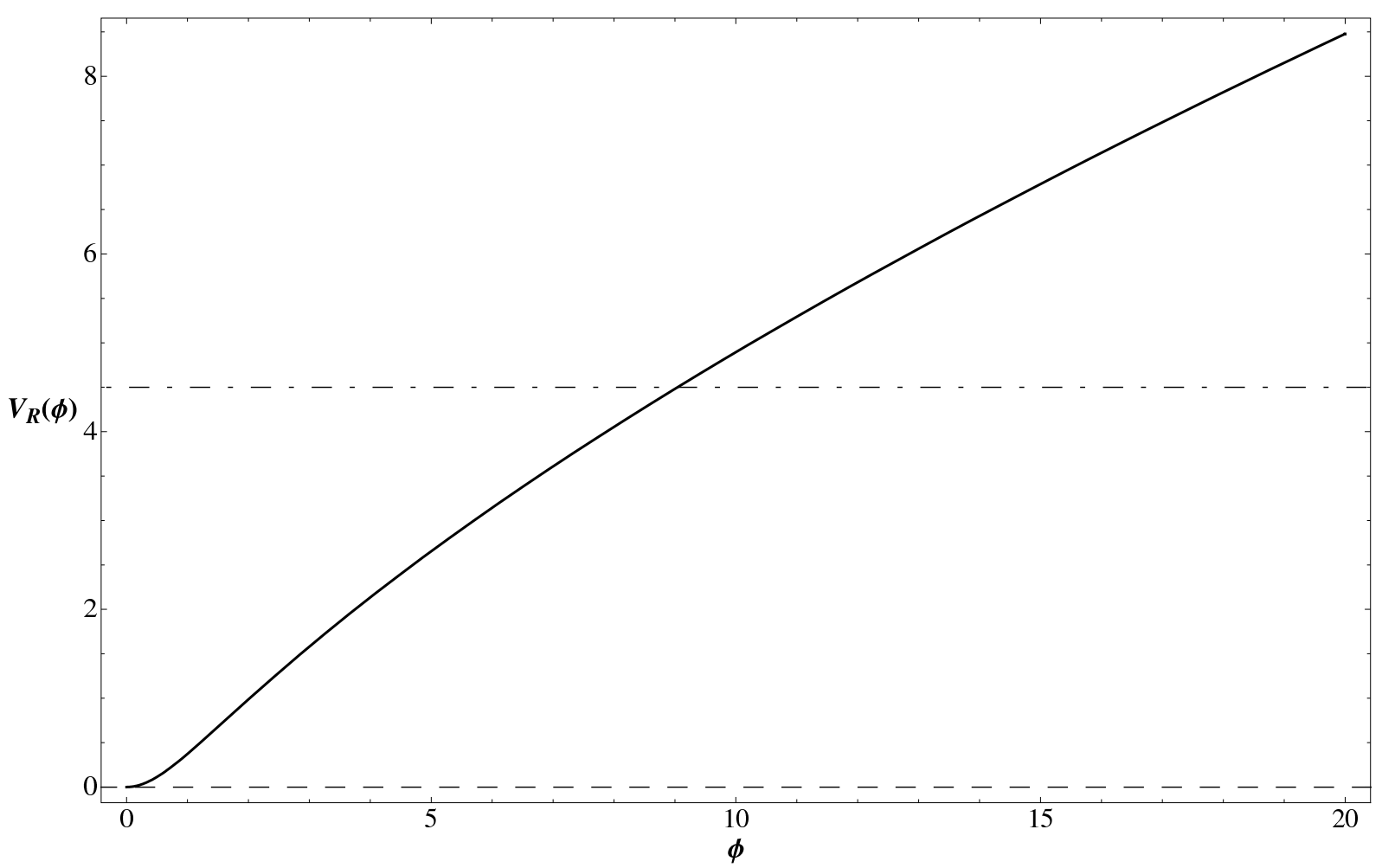

Figure 2; Solid black: The potential $V_{\mathcal{R}}(\phi)$ in arbitrary units for the canonically normalized field $\phi$ (in Planck units) corresponding to the D4-brane position $u_{1}$. The horizontal (dashdot) line indicates the scale of the moduli potential, and in the case displayed here the model is minimally tuned to avoid destabilizing the moduli at a distance in field space at about $\phi \simeq 9 M_{\mathrm{P}}$ which is the minimal field range necessary to get 60 efolds of slow-roll inflation. This corresponds to a minimally tuned anisotropy parameter $\beta \sim 0.04$ (see below).

One can clearly see in Fig. 2 that there is a regime $V_{\mathcal{R}}(\phi) \propto \phi^{2}$ close to the origin and that at large super-Planckian field values the potential grows slower than $\phi$. One can determine the behaviour of $V_{\mathcal{R}}(\phi)$ in these two regimes analytically by appropriately expanding eq. (2.12) or equivalently the square root in eq. (2.10) and then determining the canonically normalized field $\phi$ from that expansion.

We find that for

$$
u_{1}<u_{1, \mathrm{crit}} \sim \frac{1}{M} \sqrt{\beta}\left(\frac{L}{L_{x}}\right)^{3 / 2}
$$

the action eq. (2.10) expands as

$$
S_{\mathrm{D} 4}=\frac{\sqrt{\beta} L_{u}}{(2 \pi)^{4} g_{s} \alpha^{\prime 2}} \int d^{4} x \sqrt{-g_{4}}\left(\frac{L_{u}^{2}}{\beta} \alpha^{\prime} \frac{\dot{u}_{1}^{2}}{2}-\frac{L_{x}^{2} M^{2}}{2 \beta L_{u}^{2}} u_{1}^{2}\right) .
$$


This becomes canonically normalized for

$$
\phi=\frac{L_{u}^{3 / 2}}{(2 \pi)^{2} \sqrt{g_{s} \alpha^{\prime}} \beta^{1 / 4}} u_{1}
$$

yielding

$$
S_{\mathrm{D} 4}=\int d^{4} x \sqrt{-g_{4}}\left(\frac{1}{2} \dot{\phi}^{2}-\frac{m^{2}}{2} \phi^{2}\right)
$$

with

$$
m^{2}=\frac{M^{2}}{\alpha^{\prime}} \frac{L_{x}^{4}}{L^{6}}
$$

The boundary value $u_{1 \text {,crit }}$ where this expansion breaks down corresponds to a value of $\phi$

$$
\frac{\phi_{\text {crit }}}{M_{\mathrm{P}}} \sim(2 \pi)^{3 / 2} \beta^{1 / 4} \frac{\sqrt{g_{s}} L^{3 / 4}}{M L_{x}^{9 / 4} \sqrt{2}}
$$

Here we have plugged in the definition of $\alpha^{\prime}$ from eq. (2.7) to express $\phi_{\text {crit }}$ in $4 \mathrm{~d}$ Planck units. In order to determine from (2.20) whether (and under what conditions) the $m^{2} \phi^{2}$ behavior persists into the regime of $\phi_{\text {crit }}>M_{\mathrm{P}}$, one needs a concrete stabilization mechanism for $g_{s}, L, L_{x}$, and $\beta$. We will see in the construction [4] that $\phi_{\text {crit }}$ is sub-Planckian parametrically as we take appropriate flux quantum numbers large, while maintaining the stabilization of the moduli.

In fact, we can derive this conclusion - that the $m^{2} \phi^{2}$ regime of the potential (2.14) never applies self-consistently for super-Planckian field range - in a more general way. It follows from the requirement that our inflaton potential energy not exceed the scale of the potential energy $\mathcal{U}_{\text {mod, } \mathcal{R}}$ used to stabilize the moduli. In any model on Nil manifolds, the contribution to the moduli potential coming from the negative scalar curvature $\mathcal{R}$ of the compactification is of the order

$$
\mathcal{U}_{\text {mod, } \mathcal{R}} \sim M_{\mathrm{P}}^{4} \frac{(2 \pi)^{7}}{4} g^{2} \frac{M^{2} L_{x}^{4}}{L^{6}}
$$

Meanwhile, the potential $m^{2} \phi^{2}$ from $(2.18)(2.19)$ is

$$
V_{\mathcal{R}}\left(\phi<\phi_{\text {crit }}\right)=\frac{1}{2} m^{2} \phi^{2} \sim\left(\frac{\phi}{M_{\mathrm{P}}}\right)^{2} M_{\mathrm{P}}^{4} \frac{(2 \pi)^{7}}{4} g^{2} \frac{M^{2} L_{x}^{4}}{L^{6}} \sim\left(\frac{\phi}{M_{\mathrm{P}}}\right)^{2} \mathcal{U}_{\text {mod, }, \mathcal{R}}
$$

From this we see that the requirement of consistency with moduli stabilization prevents a super-Planckian field range for $\phi$ within the $m^{2} \phi^{2}$ regime of the potential (2.14). This rules out single-field slow-roll inflation in the $m^{2} \phi^{2}$ regime of our potential - the slow roll parameters $\epsilon=\frac{M_{\mathrm{P}}^{2}}{2}\left(\frac{V^{\prime}}{V}\right)^{2}$ and $\eta=M_{\mathrm{P}}^{2} \frac{V^{\prime \prime}}{V}$ are at least of order 1 for $\phi \leq M_{\mathrm{P}}$. Of course this does not rule out the possibility in general -for example, with a higher-scale moduli potential, arising in the less supersymmetric constructions among those reviewed in [16], it may be possible to accommodate $m^{2} \phi^{2}$ inflation in a different setup. 


\subsubsection{Sol manifolds}

Before continuing with our main example, let us briefly mention a similar result for Sol manifolds. Sol 3-manifolds constitute a more general class of twisted tori where the $S L(2, Z)$ transformation made in going around the base circle is more generic than a $\tau \rightarrow \tau+M$ transformation. These arise by compactifying the sol geometry

$$
d s^{2}=L_{1}^{2} d x_{1}^{2} e^{2 z}+L_{2}^{2} d x_{2}^{2} e^{-2 z}+L_{z}^{2} d z^{2}
$$

In this case, the curvature is $-2 / L_{z}^{2}$ and the volume of each 3 -manifold factor is $L^{3}=L_{1} L_{2} L_{z}$, leading to a contribution to the moduli potential of order

$$
\mathcal{U}_{\mathcal{R}, s o l} \sim M_{\mathrm{P}}^{4} \frac{(2 \pi)^{7} g_{s}^{2}}{4 L_{z}^{2} L^{6}}
$$

For a D4-brane wrapped along some generic linear combination of the $x_{1}$ and $x_{2}$ directions, and moving in $z$, we obtain from the DBI action with metric (2.23) an effective action which both for large and small $z$ has a potential of order

$$
V_{\mathcal{R}, s o l} \sim m^{2} \phi^{2} \sim \frac{1}{L_{z}^{2} \alpha^{\prime}} \phi^{2} \sim \mathcal{U}_{\mathcal{R}, \text { sol }}\left(\frac{\phi}{M_{\mathrm{P}}}\right)^{2}
$$

in terms of the canonically normalized field $\phi$, where in the last step we used (2.7).

Thus in both Nil and Sol manifolds, an $m^{2} \phi^{2}$ potential is not consistent with moduli stabilization, assuming that the curvature-induced potential is one of the leading terms in $\mathcal{U}_{\text {mod }}$. Again, it is possible that string compactifications with higher-scale potentials - such as those arising on more generic hyperbolic compactifications and/or from $D>10$ string theory - could accommodate an $m^{2} \phi^{2}$ inflationary potential, with the moduli stabilizing potential arising at a higher scale. However in absence of that, we obtain a clean parametric constraint on $m^{2} \phi^{2}$ inflation in twisted tori for which the scalar curvature contributes to moduli stabilization as in [4].

\subsubsection{Our main example: large $u_{1}$ and the $\phi^{2 / 3}$ potential}

Because we cleanly ruled out the $m^{2} \phi^{2}$ regime, let us focus on the opposite regime where $\phi \gg \phi_{\text {crit. }}$. In this case, the action eq. (2.10) expands as

$$
S_{\mathrm{D} 4}=\frac{1}{(2 \pi)^{4} g_{s} \alpha^{\prime 2}} \int d^{4} x \sqrt{-g_{4}}\left(\frac{1}{2} \frac{M L_{x} L_{u}^{2}}{\beta} \alpha^{\prime} u_{1} \dot{u}_{1}^{2}-L_{x} M u_{1}\right) .
$$

This becomes canonically normalized for

$$
\phi=\frac{M^{1 / 2} L_{u} L_{x}^{1 / 2}}{6 \pi^{2} \sqrt{g_{s} \alpha^{\prime}} \beta^{1 / 2}} u_{1}^{3 / 2}
$$


yielding

$$
S_{\mathrm{D} 4}=\int d^{4} x \sqrt{-g_{4}}\left(\frac{1}{2} \dot{\phi}^{2}-\mu^{10 / 3} \phi^{2 / 3}\right)
$$

with

$$
\mu^{10 / 3}=\left(\frac{3}{2}\right)^{2 / 3}(2 \pi)^{-8 / 3} \frac{M^{2 / 3} \beta^{1 / 3}}{\alpha^{\prime 5 / 3} g_{s}^{2 / 3}} \frac{L_{x}}{L}
$$

Altogether, we see that for $\phi \gg \phi_{\text {crit }}$ we have a potential for $\phi$ (coming from the curvature of the compactification which affects the D4-brane action) given by a fractional power

$$
V_{\mathcal{R}}(\phi) \propto \phi^{p}, p=2 / 3
$$

Single-field slow roll inflation based on any power law potential with power $p$ of order 1 requires a super-Planckian field range in order to suppress the slow-roll parameters $\epsilon=$ $\frac{M_{\mathrm{P}}^{2}}{2}\left(\frac{V^{\prime}}{V}\right)^{2}$ and $\eta=M_{\mathrm{P}}^{2} \frac{V^{\prime \prime}}{V}$. So far, our system thus provides a candidate for large field chaotic inflation with a fractional power-law potential 8

There are many self-consistency conditions and observational constraints which must now be imposed in order to assess this possibility. This will occupy the bulk of the remainder of the paper. We will find a reasonably natural viable regime where (2.30) seems to apply to good approximation, noting a few subtleties along the way. We will then review the observational predictions of single-field slow roll inflation governed by our power-law potential (2.30). Before turning to that, let us pause to note another version of this mechanism, which gives a different power-law potential in a similar way.

\subsubsection{A potential variant with $\tilde{V}(\tilde{\phi}) \propto \tilde{\phi}^{2 / 5}$}

In our product of two Nil 3-manifolds, we could consider a D4-brane wrapped on say the $u_{2}-\tilde{u}_{2}$ direction, while moving in a linear combination $u_{B}$ of $u_{1}, \tilde{u}_{1}$, and $u_{2}+\tilde{u}_{2}$ directions. In this case, the term $L_{x}^{2} M^{2}\left(u_{1}^{2} d u_{2}^{2}+\tilde{u}_{1}^{2} d \tilde{u}_{2}^{2}\right)$ in the metric would lead to a contribution to the kinetic energy for $u_{B}$ of the form

$$
\tilde{\mathcal{L}}_{k i n} \sim \cdots+\frac{1}{(2 \pi)^{4} g_{s} \alpha^{\prime 2}} \sqrt{\beta L_{u}^{2}+L_{x}^{2} M^{2} u_{B}^{2}}\left(L_{x}^{2} M^{2} u_{B}^{2} \dot{u}_{B}^{2}\right) .
$$

In this case, for large $u_{B}$ the canonically normalized field $\tilde{\phi}$ would satisfy (using the analogue of (2.11) $) u_{B} \propto \tilde{\phi}^{2 / 5}$, leading to a potential $\tilde{V}(\tilde{\phi}) \propto \tilde{\phi}^{2 / 5}$. We will continue to analyze the case with the $\phi^{2 / 3}$ potential in detail; the $\tilde{\phi}^{2 / 5}$ case can be analyzed similarly.

${ }^{8}$ The term "chaotic inflation" follows the title of the work [5] which introduced the first example. But see 19 for an analysis of the ambiguity in terminology which has resulted in the literature, since this term may refer only to the assumption of chaotic initial conditions. We cannot implement the suggestion of [19] and call our model "inflation with an unbounded potential" simply because the moduli-stabilizing potential will always cut it off at some point in field space. 


\subsection{Consistency with Moduli Stabilization and Background Geometry}

In this subsection, we will analyze several basic self-consistency criteria. First, we will impose the condition that the inflaton potential energy not destabilize the moduli. Next, we will show that the small $\phi$-dependent shifts in moduli which do arise do not destabilize the inflaton trajectory. Finally, we will analyze and bound the back reaction of our D4-brane in the ten-dimensional description.

\subsubsection{Condition $V_{\mathcal{R}}(\phi)<\mathcal{U}_{\text {mod, } \mathcal{R}}$}

A crucial first condition for the above mechanism of large-field inflation to succeed in concrete model is that the process of inflation at $\phi>M_{\mathrm{P}}$ not destabilize the moduli (a condition which immediately rules out the $m^{2} \phi^{2}$ regime of our potential, as discussed above). The moduli potential or, more precisely, the height of the barriers protecting the moduli $L, L_{x}, L_{u}$ from run-away scales as indicated in (2.21) [4].9 Introducing a source of potential energy which dominates over those used in [4] would generically remove the local de Sitter minimum found there. Thus, the most basic condition for preserving moduli stabilization is

$$
V_{\mathcal{R}}(\phi)<\mathcal{U}_{\text {mod, } \mathcal{R}} \text { for : } \phi<\phi_{\max } \text {. }
$$

Applying this condition now to the $\phi^{2 / 3}$ regime applicable for $\phi \gg \phi_{\text {crit }}$, we can start by determining whether observationally viable large field chaotic inflation can coexist with moduli stabilization at this basic level.

We find

$$
\phi_{\max } \sim M_{\mathrm{P}}(2 \pi)^{21 / 2} \frac{M_{\mathrm{P}}^{5}}{\mu^{5}} \frac{M^{3} g^{3} L_{x}^{6}}{8 L^{9}} .
$$

We note that as $\mu^{10 / 3} \propto \beta^{1 / 3}$ this implies that in this regime we have

$$
\phi_{\max } \propto \beta^{-1 / 2},
$$

suggesting that $\beta \ll 1$ may provide a parametric regime where $\phi_{\max }$ grows large - this is intuitive since suppressing $\beta$ corresponds to shrinking the cycle wrapped by our D4brane, while also expanding the direction $u_{1}+\tilde{u}_{1}$ in which it moves. However, we will find compensating constraints on $\beta$ which prevent us from tuning it to be arbitrarily small.

\footnotetext{
${ }^{9}$ We suppress dependence on the angular moduli discussed in [4. These angles have positive mass squareds in the rectangular configuration we are considering, at fixed $L, L_{x}$, and $\beta$. However, we have not explicitly computed the off-diagonal elements in the mass matrix between the angular moduli and the others. If these are large enough, tachyonic directions could develop; this depends on order 1 coefficients in the potential and its derivatives. A cursory look at the terms which contribute to the mass matrix suggests that these off-diagonal elements are likely to be tuneable to be numerically smaller than the diagonal contributions. In particular, there are many ingredients one can add which are of the same order or less than the original moduli potential, and which push on the angles in different directions. However this - and the similar problem of determining the off-diagonal elements in the mass matrix mixing the lightest KK modes with the moduli in the simplest version of the setup described in [4] - remains to be worked out explicitly.
} 
The value of $\phi_{\max }$ depends on the stabilized values of the moduli. It is now worth plugging in the results for the moduli in terms of flux quantum numbers in the construction [4], type IIA on a product $\mathcal{N}=\mathcal{N}_{3} \times \tilde{\mathcal{N}}_{3}$ of Nil three-manifolds with six-form flux $F_{6}$ satisfying the quantization condition $\int_{\mathcal{N}} F_{6}=K$, with

$$
K=\frac{1}{\sqrt{2}}(2 \pi)^{5} f_{6} \sim 7 \times 10^{3} f_{6}
$$

in terms of the integer flux quantum number $f_{6}$. In this construction, the VEVs of the moduli in their dS minimum scale in terms of the basic topological and flux quantum numbers $M, K$ as

$$
L=c_{L} \cdot K^{1 / 6} \quad, \quad L_{x}=\frac{c_{L_{x}}}{M^{1 / 2}} \quad, \quad g=\frac{\hat{g}}{K}
$$

$c_{L}, c_{L_{x}}$ here are numerically constant, which for a generic Nil manifold compactification will be of $\mathcal{O}(1)$.

In terms of these quantities, we find for $\phi_{\text {crit }}$ and $\phi_{\max }$

$$
\begin{aligned}
\frac{\phi_{\text {crit }}}{M_{\mathrm{P}}} & \sim(2 \pi)^{3 / 2} \beta^{1 / 4} \hat{g}^{1 / 2}\left(\frac{M}{K}\right)^{1 / 8}\left(\frac{c_{L}}{c_{L_{x}}}\right)^{9 / 4} \\
\frac{\phi_{\max }}{M_{\mathrm{P}}} & \sim \frac{1}{3} \beta^{-1 / 2}\left(\frac{K}{M}\right)^{1 / 4}\left(\frac{c_{L}}{c_{L_{x}}}\right)^{-9 / 2} \frac{1}{(2 \pi)^{3} \hat{g}}
\end{aligned}
$$

These relations go in the right direction for our mechanism, as the ratio $M / K$ is much smaller than one in even the simplest version of the construction [4]. In some ways, this construction becomes better controlled (in the sense that the KK mass scale is parametrically larger than the moduli mass scale) in the regime $K / M \rightarrow \infty$, though arranging this requires extra ingredients which complicate the analysis. In this regime, $10 \phi_{\text {crit }}$ decreases and $\phi_{\max }$ increases. In all versions, we find ourselves squarely in the regime $V_{\mathcal{R}} \propto \phi^{2 / 3}$. See Fig. 2 for a graphical depiction of the candidate inflaton potential and the moduli potential, showing the finite but super-Planckian range of $\phi$ consistent with moduli stabilization.

Since we are including the degree of freedom $\beta$, we must also keep track of its effects on the moduli stabilization mechanism. The ingredients which contribute leading terms to the moduli potential must be arranged so as to produce the value of $\beta \sim L_{2} / L_{1}$ used for inflation. We will be led to consider, for example, KK fivebranes on the cycle generated by the translations $t_{u_{1}} t_{\tilde{u}_{1}} t_{u_{2}}^{1 / b} t_{\tilde{u}_{2}}^{1 / b}$, with $b$ taken to be of order $\beta$ so as to stabilize $L_{u_{2}} / L_{u_{1}}$ at the value $\beta$. This introduces a factor of $1 / \sqrt{\beta}$ in the corresponding KK5-brane contribution to the potential energy relative to the expression in eq. (3.22) of [4] (which pertained to the isotropic arrangement $L_{u_{1}}=L_{u_{2}}$ ). Now we have

$$
\mathcal{U}_{K K 5} \propto \frac{1}{\sqrt{\beta}} M_{\mathrm{P}}^{4} g^{2} \frac{M L_{x}^{5 / 2}}{L^{9 / 2}}
$$

\footnotetext{
${ }^{10}$ and also for $\beta \ll 1$, which is limited by competing effects we will soon discuss
} 
Because of this, $\beta$ cannot be reduced arbitrarily, since the terms in $\mathcal{U}_{\text {mod }}$ were already all of the same order in the construction [4]. In a sample example to be discussed below, we will find that we need only a modest tune of $\beta \sim 0.04$. However, eternal inflation would require a much stronger tune of $\beta$, which would need to be reevaluated vis a vis moduli stabilization.

Let us finally note here that the condition eq. (2.32) necessary for avoiding moduli destabilization implies the same relationship between the inflationary Hubble parameter and the gravitino mass

$$
H_{\mathrm{inf}} \leq m_{3 / 2}
$$

as discussed in the context of the KKLT-style IIB de Sitter vacua in [25]. In our present case, this follows from the fact that the scale of supersymmetry breaking in a typical Nil manifold compactification is at or above the curvature scale: $m_{3 / 2} \geq \sqrt{\mathcal{R}}$. So since $\mathcal{U}_{\text {mod, } \mathcal{R}} \sim M_{\mathrm{P}}^{2}(-\mathcal{R})$, (2.32) implies

$$
V_{\mathcal{R}} \sim M_{\mathrm{P}}^{2} H_{\mathrm{inf}}^{2} \leq M_{\mathrm{P}}^{2} m_{3 / 2}^{2}
$$

\subsubsection{Moduli Shifts and the Inflaton potential}

The moduli are not destabilized by our additional contribution $V_{\mathcal{R}}$ to the potential energy, but they do shift slightly due to its presence. Let us analyze the effect of this on our candidate inflaton potential. Schematically the potential is of the form

$$
\mathcal{U}_{\text {tot }} \sim \mathcal{U}_{\text {mod }}\left(L e^{\frac{\sigma}{M_{\mathrm{P}}}}, \ldots\right)+V_{\mathcal{R}}\left(\phi, L e^{\frac{\sigma}{M_{\mathrm{P}}}}, \ldots\right)
$$

where we keep track of the dependence on both the inflaton $\phi$ and on the moduli (represented by $\left.L e^{\frac{\sigma}{M_{\mathrm{P}}}}, \ldots\right)$. Here $L$ denotes the stabilized value of the modulus in the absence of the inflaton potential, and $\sigma$ is the canonically normalized scalar field describing the deformation of the modulus away from this value.

In particular, $\left.\partial_{\sigma}\right|_{\sigma=0} \mathcal{U}=0$. Taylor expanding the full potential about $\sigma=0$, we obtain (suppressing the dependence on the other moduli ..., which work similarly)

$$
\mathcal{U}_{\text {tot }} \sim \mathcal{U}_{\text {mod }}(L)+\frac{1}{2} \partial_{\sigma}^{2} \mathcal{U}_{\text {mod }}(L) \frac{\sigma^{2}}{M_{\mathrm{P}}^{2}}+V_{\mathcal{R}}(\phi, L)+\partial_{\sigma} V_{\mathcal{R}}(\phi, L) \frac{\sigma}{M_{\mathrm{P}}}+\frac{1}{2} \partial_{\sigma}^{2} V_{\mathcal{R}}(\phi, L) \frac{\sigma^{2}}{M_{\mathrm{P}}^{2}}
$$

Now $V_{\mathcal{R}}$ and each individual term in the moduli potential is proportional to a power of $L e^{\frac{\sigma}{M_{\mathrm{P}}}}$. So the derivatives of each term with respect to $\sigma$ scale the same parametrically - they depends on the same powers of $L, \ldots$ - as the term itself. Note that although the de Sitter minimum of the moduli potential is tuned to lie near zero vacuum energy: $\mathcal{U}_{\text {mod }}(L) \ll \mathcal{U}_{\text {mod, } \mathcal{R}}(L)$, the second derivative $\partial_{\sigma}^{2} \mathcal{U}_{\text {mod }}(L)$ is not tuned to be small. It is of the same order as a typical term in the moduli potential, $\sim \mathcal{U}_{\text {mod, } \mathcal{R}}$.

Putting this together, we have from (2.43) a small shift in the moduli

$$
\frac{\sigma}{M_{\mathrm{P}}} \sim \frac{\partial_{\sigma} V_{\mathcal{R}}(\phi, L)}{\partial_{\sigma}^{2} \mathcal{U}_{\text {mod }}(L)+\partial_{\sigma}^{2} V_{\mathcal{R}}(\phi, L)} \sim \frac{V_{\mathcal{R}}(\phi, L)}{\mathcal{U}_{\text {mod }, \mathcal{R}}(L)}
$$


Plugging this back into the potential (2.43) we have (for some order one constant $c_{L \phi}$ )

$$
\mathcal{U}_{\text {tot }} \sim \mathcal{U}_{\text {mod }}(L)+V_{\mathcal{R}}(\phi, L)+c_{L \phi} \frac{V_{\mathcal{R}}(\phi, L)^{2}}{\mathcal{U}_{\text {mod, } \mathcal{R}}(L)}
$$

This gives a small change in the functional form of the inflaton potential, shifting the slow-roll parameter $\eta=M_{\mathrm{P}}^{2} \frac{\partial_{\phi}^{2} V}{V}$ by a term of order $\leq \eta$ itself

$$
\Delta \eta \sim \eta \frac{V_{\mathcal{R}}(\phi, L)}{\mathcal{U}_{\text {mod, } \mathcal{R}}}
$$

Thus these small shifts of the moduli do not destabilize inflation, and only for the case $V_{\mathcal{R}} \sim U_{\text {mod, } \mathcal{R}}$ saturating $(2.32)$ could they contribute significantly to the tilt of the power spectrum (which depends on $\eta$ in a way we review below).

\subsubsection{Back reaction of branes in $10 d$}

Deep in our regime where $V_{\mathcal{R}}(\phi) \propto \phi^{2 / 3}$, the D4-brane wrapped on the (M,1) cycle of the torus traced out by $x^{\prime}, u_{2}$ constitutes to good approximation a set of $M u_{1}$ branes wrapped in the $x$ direction, spaced evenly in the $u_{2}$ direction. As mentioned above in $\$ 2$, in this sense we are considering a multiply wrapped brane. As discussed in previous works such as [15], multiple and/or wrapped branes - which help extend the field range even for Calabi-Yau compactifications - can lead to significant back reaction, and we should check this in our case. In our discussion of loop corrections below, we will also need the number of species introduced by our effectively multiply wrapped brane, in assessing the strength of e.g. the renormalization of $M_{\mathrm{P}}[12$.

First, note that using the fact from (2.26) that $V_{\mathcal{R}} \sim \frac{L_{x} M u_{1}}{(2 \pi)^{4} g_{s}\left(\alpha^{\prime}\right)^{2}}$, and using (2.7) and (2.21), we can express the number of windings $M u_{1}$ of our brane around the $x$ direction as

$$
N_{w} \equiv M u_{1} \sim \frac{V_{\mathcal{R}}}{\mathcal{U}_{m o d, \mathcal{R}}} \frac{2 L_{x}^{3} M^{2}}{(2 \pi)^{3} g_{s}}
$$

We must check whether this multiply wound brane still constitutes a probe of the geometry, as we have assumed in writing its DBI action (2.9).

Let us dimensionally reduce on the $u_{2}, x, \tilde{x}$ directions, and determine the core size of our wrapped D4 brane in the remaining $\tilde{u}_{2}, u_{1}$, and $\tilde{u}_{1}$ directions. We can estimate this as follows. The gravitational potential in the 3 directions $\tilde{u}_{2}, u_{1}, \tilde{u}_{1}$ is given by

$$
\Phi_{\text {grav }} \sim \frac{G_{7} V_{\mathcal{R}}}{|\vec{r}|}
$$

where $G_{7} \sim(2 \pi)^{4} \frac{g_{s}^{2}\left(\alpha^{\prime}\right)^{5 / 2}}{L_{x}^{2} L_{u_{2}}}$ is the Newton constant in the remaining seven dimensions after the dimensional reduction along $u_{2}, x, \tilde{x}$, and $|\vec{r}|$ is the proper distance from the source D4-brane. 
Here we work in the flat space approximation (which will be valid if we arrange that the core size is smaller than the curvature radii), using the BPS formula for the brane tensions which is a good approximation for low curvature. From (2.48) we can read off the the core size of our brane:

$$
r_{c} \sim G_{7} V_{\mathcal{R}}
$$

If the core size $r_{c}$ is smaller than $L_{u_{1}}$ and the curvature radius $r_{\mathcal{R}}$, then the brane is a good probe as far as motion in the $u_{1}$ direction goes (as we have been assuming). First, note that

$$
\frac{r_{c}}{L_{u_{2}} \sqrt{\alpha^{\prime}}} \sim \frac{V_{\mathcal{R}}}{\mathcal{U}_{\text {mod, } \mathcal{R}}} \frac{L_{x}^{3} M^{2}}{\beta L^{3}(2 \pi)^{3}} \sim \frac{V_{\mathcal{R}}}{\mathcal{U}_{\text {mod, } \mathcal{R}}} \frac{1}{\beta(2 \pi)^{3}} \sqrt{\frac{M}{K}}
$$

Correspondingly,

$$
\frac{r_{c}}{L_{u_{1}} \sqrt{\alpha^{\prime}}} \sim \frac{V_{\mathcal{R}}}{\mathcal{U}_{\text {mod }, \mathcal{R}}} \frac{L_{x}^{3} M^{2}}{L^{3}(2 \pi)^{7}} \sim \frac{V_{\mathcal{R}}}{\mathcal{U}_{\text {mod, }, \mathcal{R}}} \frac{1}{(2 \pi)^{7}} \sqrt{\frac{M}{K}}
$$

In our regime of interest, we will have $K \gg M$ and $\beta \ll 1$, with the ratio $\frac{V_{\mathcal{R}}}{\mathcal{U}_{\text {mod, } \mathcal{R}}} \propto \beta^{1 / 3}$. The expression (2.50) shows that we cannot decrease $\beta$ arbitrarily without causing the core size of our wrapped D4-brane to exceed $L_{u_{2}}$, requiring a new analysis of the core size in the remaining $u_{1}, \tilde{u}_{1}$ directions. (That is, the expressions (2.50), (2.51) are only valid if both are small.) However we will find that there is a substantial window in which the ratios (2.50), (2.51) are highly suppressed. This also implies that the core size is much smaller than the curvature radii in our space and the probe approximation is valid (the curvature invariants here, $\mathcal{N}_{3}$ being three dimensional, are $R, R^{m n} R_{m n}$, $\operatorname{det} R / \operatorname{det} g$, and thus we get all three curvature radii to be of the same order: $R^{-1 / 2},\left(R^{m n} R_{m n}\right)^{-1 / 4},(\operatorname{det} R / \operatorname{det} g)^{-1 / 6} \sim$ $\left.L_{u}^{2} /\left(M L_{x}\right) \gg L_{u_{2}}\right)$.

\subsection{Basic Observational Constraints: e-foldings and power spectrum}

We must satisfy two basic requirements for the observational viability of the model - conditions on the number of e-foldings and the normalization of the scalar power spectrum. At fixed $\beta$ these two conditions fix $M$ and $K$, so it will prove useful to retain the independent parameter $\beta$. It is worth emphasizing that because of the numerical factors in (2.35), a large value of $K$ need not correspond to a large value of the input flux quantum number $f_{6}$.

For any model with a single stage of inflation to be observationally viable, it must produce at least some 60 efolds of slow-roll inflation before the process exits to the minimum of the potential in order to solve the isotropy, homogeneity and entropy problems of standard hot big bang cosmology [1, 26]. For large field chaotic inflation with a power-law potential $V(\phi) \propto \phi^{p}$ the field $\phi$ has to start at a value of

$$
\phi_{N_{e}}=\sqrt{2 p N_{e}} M_{\mathrm{P}}
$$


to generate $N_{e}$ efolds of slow-roll inflation before inflation ends. The number of efolds is determined in slow-roll by

$$
N_{e}=\int_{t_{N_{e}}}^{t_{\mathrm{exit}}} H d t \simeq \int_{M_{\mathrm{P}}}^{\phi_{N_{e}}} \frac{d \phi}{M_{\mathrm{P}}^{2}} \frac{V}{V^{\prime}}=\frac{1}{2 p}\left(\frac{\phi_{N_{e}}^{2}}{M_{\mathrm{P}}^{2}}-1\right)
$$

Two basic observational conditions for the viability of our mechanism are (i) Obtaining $\phi_{\max }$ larger than $\phi_{60}=2 \sqrt{N_{e} / 3} M_{\mathrm{P}} \simeq 9 M_{\mathrm{P}}$ at $N_{e}=60$ in our regime where $V_{\mathcal{R}}(\phi)=$ $\mu^{10 / 3} \phi^{2 / 3}$, while also (ii) obtaining the appropriate scale of the density perturbations at $N_{e}=60$ efolds before the end of inflation.

This latter criterion is that we must generate a level of scalar curvature perturbation $\left.\Delta_{\mathcal{R}}\right|_{60} \simeq 5.4 \times 10^{-5}$. In our system,

$$
\left.\Delta_{\mathcal{R}}\right|_{N_{e}}=\left.\sqrt{\frac{1}{12 \pi^{2}} \frac{V^{3}}{M_{\mathrm{P}}^{6} V^{\prime 2}}}\right|_{N_{e}}=\frac{(4 / 3)^{1 / 6}}{2 \pi} N_{e}^{2 / 3} \frac{\mu^{5 / 3}}{M_{\mathrm{P}}^{5 / 3}}
$$

This depends on a combination of powers of $\beta$ and $L, L_{x}$ which is independent of the one appearing in eq. (2.33). We will shortly write this in terms of the physical quantities $M$ and $K$ in the construction [4]. This will leave a candidate window for inflation - consistent with moduli stabilization, $N_{e} \simeq 60$, and $\left.\Delta_{\mathcal{R}}\right|_{60} \simeq 5.4 \times 10^{-5}$ - obtained with only modest tuning of the parameters. Then we will analyze the problem of controlling all $\mathcal{O}\left(10^{-2}\right)$ contributions to $\epsilon$ and $\eta$ arising our string compactification. Before turning to that, we pause to also indicate the conditions for eternal inflation in our background, which would involve even greater super-Planckian excursions in field space.

\subsection{Conditions for Eternal inflation}

The scalar curvature perturbations $\Delta_{\mathcal{R}}$ and in turn the primordial density perturbations are both generated from the quantum fluctuations of scalar fields in a de Sitter background

$$
\sqrt{\left\langle\delta \phi^{2}\right\rangle_{q}}=\frac{H}{2 \pi}
$$

They begin to dominate the classical slow-roll motion once we have $\Delta_{\mathcal{R}} \gtrsim 1$. If this is case then inflation in that regime never ends but is started over and again, rendering inflation in the global space-time eternal to the future. The boundary of eternal inflation, $\phi_{*}$, can thus be determined from

$$
\left.\frac{1}{M_{\mathrm{P}}^{3}} \frac{V^{3 / 2}}{V^{\prime}}\right|_{\phi_{*}} \gtrsim \sqrt{12 \pi^{2}}
$$

For a power law potential, the value of $\phi_{*}$ is thus fixed once the curvature perturbation at 60 efolds before the end of inflation has been normalized to the COBE value: E.g. in the 
$\mu^{10 / 3} \phi^{2 / 3}$ under study here the ratio $V / \epsilon$ is controlled by $\mu$ whose value is fixed by the COBE normalization to be

$$
\mu_{\text {obs. }} \simeq 1.6 \times 10^{-3}
$$

Now $\phi_{*}$ is fixed once $\mu$ is observationally determined, while $\phi_{\max }$ can be adjusted to some extent by decreasing $\beta$, though $\beta$ cannot be reduced arbitrarily without introducing problematic back reaction (2.50) and complicating moduli stabilization (2.39). When it applies, eternal inflation may considerably mitigate the problem of initial conditions for inflation.

\subsection{Specific results in a concrete Nil manifold construction of dS vacua}

The results of the last sections concerning the appearance of a candidate large field chaotic inflation model with a $\mu^{10 / 3} \phi^{2 / 3}$-potential for the D4-brane motion apply rather generally in compactifications of type IIA string theory to $4 \mathrm{~d}$ on Nil manifolds.

However, the parameters of the model depend on the moduli VEVs $L, L_{x}, L_{u}$, and $\beta$, which are determined in terms of topological, brane, and flux quantum numbers in any concrete compactification. To assess the viability of our mechanism in a concrete model, we therefore include in this section the formulas for our model parameters in terms of these discrete quantum numbers in the construction [4] on a product $\mathcal{N}_{3} \times \tilde{\mathcal{N}}_{3}$ of Nil three-manifolds. It was shown there that the VEVs of the potentially runaway moduli in their $\mathrm{dS}$ minimum scale in terms of the brane and flux quantum numbers $M, K$ as

$$
L=c_{L} \cdot K^{1 / 6} \quad, \quad L_{x}=\frac{c_{L_{x}}}{M^{1 / 2}} \quad, \quad g=\frac{\hat{g}}{K} \quad .
$$

$c_{L}, c_{L_{x}}$ here are numerical constants, and for a generic Nil manifold compactification will be of $\mathcal{O}(1)$. For the minimal setup described in [4], we estimate their values as

$$
c_{L} \simeq\left(56 \pi^{2}\right)^{1 / 12} \simeq 1.7 \quad, \quad c_{L_{x}}=3^{1 / 8} 2^{5 / 4} \pi \simeq 8.6 \quad, \quad \hat{g}=\sqrt{\frac{2}{7}}(6+\sqrt{3}) \pi^{2} \simeq 41
$$

We will include these specific numbers to get a feel for the magnitudes of the parameters that might arise in a specific model realizing our mechanism - however we emphasize that there are many variants of the construction which will shift the values (2.58), and in the expression for our estimate of $\hat{g}$ we neglected the KK5-brane contribution for simplicity (this would kick up $\hat{g}$ slightly). In terms of $L^{3}=L_{u}^{2} L_{x}$ and $L_{x}$ we have then

$$
L_{u}=\frac{c_{L}^{3 / 2}}{c_{L_{x}}^{1 / 2}}(K M)^{1 / 4} \simeq 0.75 \times(K M)^{1 / 4}
$$

We see that there is a controlled regime with $K \gg M>1$ for which $L, L_{u} \gg L_{x}$ and $L, L_{u} \gg$ 1. In particular, we will be interested in a regime which satisfies $K \gg M$ numerically, without 
taking a limit where $K / M$ becomes parametrically large; a sample numerical solution of this kind was described in $\S 3.8$ of [4]. This setup is simplest in that it contains the fewest ingredients, but is subject to several subtleties noted in [4: the KK and moduli mass matrices are positive definite in themselves, but the question of whether unstable directions might arise due to their mixing has not been analyzed in detail.

We can now plug these results into the general formulas above for the values $\phi_{\text {crit }}, \phi_{\max }$ and $\phi_{*}$ (describing the boundaries to the $m^{2} \phi^{2}$-regime, the destabilization of the moduli, and the regime of eternal inflation, respectively) as well as into the result for the curvature perturbation. We arrive then at

$$
\begin{aligned}
\frac{\phi_{\text {crit }}}{M_{\mathrm{P}}} & \sim(2 \pi)^{3 / 2} \gamma^{-1 / 2} \frac{\hat{g}^{1 / 2}}{\sqrt{2}}\left(\frac{c_{L}}{c_{L_{x}}}\right)^{9 / 4} \\
\frac{\phi_{\max }}{M_{\mathrm{P}}} & \sim \frac{1}{3} \frac{\gamma}{\hat{g}(2 \pi)^{3}}\left(\frac{c_{L}}{c_{L_{x}}}\right)^{-9 / 2} \\
\frac{\phi_{*}}{M_{\mathrm{P}}} & \sim K^{9 / 8} \gamma^{1 / 4}
\end{aligned}
$$

and

$$
\left.\Delta_{\mathcal{R}}\right|_{N} \sim 60^{2 / 3} \frac{(2 \pi)^{7 / 2}}{2^{5 / 6}} K^{-3 / 2} \gamma^{-1 / 3} \hat{g}^{4 / 3} c_{L}^{-1}\left(\frac{c_{L}}{c_{L_{x}}}\right)^{-1 / 2} .
$$

where we plugged in $N_{e}=60$. Here $\gamma$ denotes the combination

$$
\gamma \equiv \beta^{-1 / 2}\left(\frac{K}{M}\right)^{1 / 4}
$$

This is larger than 1 in our regime of interest with $\beta \lesssim 1$ and $K>M$.

Now the ratio $c_{L} / c_{L_{x}} \simeq 0.2$ in the concrete construction is consistent with our parametric result that $\phi_{\text {crit }}$ tends to be small (unless $\beta \gg 1$ ), pushing us deep into the $\mu^{10 / 3} \phi^{2 / 3}$-regime of these formulae, and that $\phi_{\max } \gg M_{\mathrm{P}}$ if $\beta \lesssim 1$ and $K \gg M$.

More explicitly we can see that we need, firstly, $\gamma \gtrsim 190$ for $\phi_{\max }>9 M_{\mathrm{P}}$ to get at least some 60 efolds of slow-roll inflation. Secondly, the observational constraint $\left.\Delta_{\mathcal{R}}\right|_{60}=$ $5.4 \times 10^{-5}$ implies $\gamma^{1 / 3} K^{3 / 2} \sim 1.9 \times 10^{10}$, and putting this together with the condition $\gamma \geq 190$ gives $K \leq 2.2 \times 10^{6}$, corresponding to a modest flux quantum number $f_{6} \leq 310$ (using $(2.35)$ ). Putting this together with (2.63) , we have that

$$
\beta M^{1 / 2} \leq 0.04
$$

Thus for $M \sim 1$, we obtain $\beta \sim 0.04$, and for $M \sim 10, \beta \sim .01$.

We note that the numbers we have obtained for $M$ and $f_{6}$ are close to those obtained in the sample numerical solution discussed in the simplest version of the construction [4] (the version without additional NS5-branes added to reduce the KK5-brane tensions). This will 
be useful for us, since additional moduli-stabilizing ingredients complicate the problem of suppressing contributions to the slow-roll parameters. We will shortly comment on the open question of parametric limits with arbitrarily small couplings and curvatures.

The number $M u_{1}$ of wrappings of our brane around the $x$ direction is now given by (2.47)

$$
N_{w}=M u_{1} \sim \frac{V_{\mathcal{R}}}{\mathcal{U}_{\text {mod }, \mathcal{R}}} \frac{1}{(2 \pi)^{3} \sqrt{2}} \frac{1}{\hat{g}}\left(\frac{c_{L_{x}}}{c_{L}}\right)^{3}(K M)^{1 / 2} \sim \frac{V_{\mathcal{R}}}{\mathcal{U}_{\text {mod }, \mathcal{R}}} 20 M^{1 / 2}
$$

and the ratio (2.50) of the core size $r_{c}$ to $L_{u_{2}} \sqrt{\alpha^{\prime}}$ is consistently extremely small.

A much stronger fine-tuning of $\beta$ (c.f. [27]) would be required to obtain eternal inflation, and would need to be analyzed with respect to the back reaction criteria discussed above.

\subsubsection{Conditions for a Parametric Effect}

We note that so far in this section, we have fixed some of our parameters using the observed COBE normalization for the scalar power spectrum. At the resulting values of $K, M$, and $\beta$ we obtain results consistent with the large-volume, weakly curved, locally supersymmetric weak- $g$ regime of the compactifications studied in [4] (subject to the same subtleties with respect to separating the KK and moduli mass scales indicated there).

At a theoretical level, one might wonder if the effect can be made "parametrically large", increasing the field range to be arbitrarily large while systematically improving the level of control so that the expansion parameters become arbitrarily small (without necessarily imposing the condition $\left.\Delta_{\mathcal{R}}\right|_{60} \simeq 5.4 \times 10^{-5}$ ).

Let us comment on this question here. Using the relations derived above, but not imposing the COBE normalization on the power spectrum, we have the conditions

$$
\left.\Delta_{\mathcal{R}}\right|_{\phi=\phi_{\max }} \sim 10^{3} \frac{\gamma}{K^{3 / 2}} \leq 1 \quad \frac{\phi_{\max }}{M_{\mathrm{P}}} \sim 0.1 \gamma \rightarrow \infty \quad \frac{r_{c}}{L_{u_{2}} \sqrt{\alpha^{\prime}}} \sim \frac{0.005}{\beta^{2} \gamma^{2}} \ll 1
$$

where as above, $\gamma=\beta^{-1 / 2}(K / M)^{1 / 4}$. From this we see firstly that in order to parametrically increase the field range, we must take $\gamma$ large. Given that, in order to prevent arbitrarily large curvature perturbations, we must increase $K$ such that $K^{3 / 2} \geq 10^{3} \gamma$.

As far as these formulae go, one could obtain such a parametric limit by taking $K$ sufficiently large (without needing to adjust the anisotropy $\beta$ ). This suppresses the coefficient $\mu^{10 / 3}$ in our potential $\mu^{10 / 3} \phi^{2 / 3}$, extending the field range within the regime where the inflationary potential is smaller than the moduli potential, and decreasing the back reaction parametrically. In the construction [4], increasing $K / M$ is in fact desirable also in order to increase the ratio of the lightest KK masses to the heaviest moduli masses, and a method for achieving this was sketched in that work. However, this method requires a significant elaboration of the construction - it involves introducing local sources of larger string coupling, such as NS5-branes, which intersect with the KK5-branes so as to lower their tension. As we 
will see in the next section, such defects generically complicate the problem of suppressing contributions to the slow-roll parameter $\eta$, though a symmetric arrangement of them might make it possible to make use of this more general construction to seek an explicit parametric limit of our large-field model. Note that it is not possible to parametrically increase the effect merely by decreasing $\beta$, since then the final condition in (2.66) - that of avoiding large back reaction from the wrapped brane - would eventually fail.

Regardless, again we note that the parameters we need for a self-consistent - and observationally accurate - model realizing our monodromy mechanism appear to be reasonable. That is, they lie in a large-radius, locally supersymmetric weakly curved, weak- $g$ regime of the Nil manifold compactification, suggesting that the model is controllable as argued in [4]. Therefore we return to our main example, and turn to a systematic analysis of the slow-roll parameters, and the corrections affecting them, in our setup.

\section{Theory Foregrounds: Systematic Analysis of Corrections}

So far we have seen that the curvature-induced potential $V_{\mathcal{R}}(\phi)$ provides a promising candidate inflaton potential. However, we must include all contributions to the full potential $V_{\text {tot }}\left(\phi, \phi_{\perp}\right)$ (as a function of $\phi$ and any other light scalar fields $\phi_{\perp}$ in the problem) which affect the slow-roll parameters

$$
\epsilon=\frac{1}{2} M_{\mathrm{P}}^{2}\left(\frac{V^{\prime}}{V}\right)^{2} \lesssim 0.01, \quad \eta=M_{\mathrm{P}}^{2} \frac{V^{\prime \prime}}{V} \lesssim 0.01
$$

at the $\mathcal{O}(0.01)$ level. The standard " $\eta$ problem", for example, can be phrased (independently of the scale of supersymmetry breaking) as the fact that a generic dimension six Plancksuppressed operator of the form

$$
V(\phi) \frac{\left(\phi-\phi_{0}\right)^{2}}{M_{\mathrm{P}}^{2}}
$$

would make an $\mathcal{O}(1)$ contribution to $\eta$.

Moreover, we must ensure (3.67) holds at all points on the inflaton trajectory (ranging over approximately 9 Planck units in field space in our $\phi^{2 / 3}$ model). A priori, for a candidate large-field model such as ours, this condition requires a functional fine tune of parameters in the effective action. In our setting, however, the monodromy of our D4-brane leads to the following simplification. Each time the D4-brane moves around the $u_{1}$ direction, it becomes heavier as it wraps a longer cycle, but it encounters the same background sources contained in the stabilized compactification. This means that as long as we take into account the longer cycle wrapped by the brane in each interval $\Delta u_{1}=1$, the methods for analyzing corrections to the potential are essentially the same in each such (sub-Planckian) interval. Thus if small-field brane inflation is controllable, similar methods may apply in the present setting to render large-field inflation controllable. 


\subsection{Contributions to $V, \epsilon$, and $\eta$ from Moduli-Stabilizing Ingredients}

Let us start by addressing the contributions to the background fields in (2.4) which arise from the ingredients in the construction [4] which go beyond the curvature of the Nil manifold already considered. As discussed in [20, 21, 22, 23, localized sources involved in moduli stabilization often lead to order one contributions to slow-roll parameters, some of which are rather subtle. For example, the interaction energy between the brane and other localized sources gets important contributions both from modes propagating directly between them and from modes propagating from one to the other by going around the compactification. In our problem, the homogeneity of the underlying Nil manifold combined with the symmetries and the extended nature of the sources of moduli stabilization will help suppress these effects. Let us start, however, by reviewing how this problem arises explicitly in our setup.

Let us begin by determining the effects of other localized defects (branes and orientifolds). In the construction [4], the compactification manifold is a product $\mathcal{N}_{3} \times \tilde{\mathcal{N}}_{3}$ of two Nil 3-manifolds of the form (2.1) with coordinates $u_{1}, u_{2}, x$ and $\tilde{u}_{1}, \tilde{u}_{2}, \tilde{x}$ respectively. It is convenient to dimensionally reduce on the $x, \tilde{x}$ directions, since these are stabilized at a small radius in the construction. Doing this, we can write schematically the general form of the interaction potential energy $\Delta V_{4, X}$ between our D4-brane and other local sources $X$ :

$$
\Delta V_{4, X}=G_{N, 8} \int d^{4} u L_{u}^{4}\left(\alpha^{\prime}\right)^{2} \int d^{4} u^{\prime} L_{u}^{4}\left(\alpha^{\prime}\right)^{2} \frac{\rho_{4}(u) \rho_{X}\left(u^{\prime}\right)}{L_{u_{1}}^{2} \alpha^{\prime}\left(\vec{u}_{1}-\vec{u}_{1}^{\prime}\right)^{2}+L_{u_{2}}^{2} \alpha^{\prime}\left(\vec{u}_{2}-\vec{u}_{2}^{\prime}\right)^{2}}
$$

where $G_{N, 8}=\Gamma(7 / 2) 2^{5} \pi^{7 / 2}\left(\alpha^{\prime}\right)^{3} g_{s}^{2} / L_{x}^{2}$ is the eight-dimensional Newton constant, and $\vec{u}_{i}$ refers to the two-component vector $u_{i}, \tilde{u}_{i}$ for each $i=1,2$. The energy densities $\rho_{4}(u)$ and $\rho_{X}\left(u^{\prime}\right)$ are delta-function localized at the positions of the sources, and proportional to the tension of the object. For the D4-brane, $\rho_{4}(u)$ is proportional $\tau_{4}=\frac{1}{(2 \pi)^{4}\left(\alpha^{\prime}\right)^{5 / 2} g_{s}}$.

Let us start with the KK5-branes in the construction since they contribute potential energy at tree level. The total KK5-brane tension is $n_{K} \tau_{K K 5}=\zeta n_{K} \frac{L_{x}^{2}}{\beta^{1 / 2}(2 \pi)^{7} g_{s}^{2}\left(\alpha^{\prime}\right)^{3}}$ (where $n_{K}$ is the number of KK5-branes, required to be a multiple of $M$ in [4], and where $\zeta$ depends on the local value $e^{\phi_{l o c}}$ of the string coupling in the region where the KK5-brane sits [4] : $\left.\zeta=g_{s}^{2} e^{-2 \phi_{l o c}}\right)$.

The interaction energy of the D4-brane with the KK5-branes depends on how they are oriented relative to each other and on the distance between them. After dimensionally reducing on the $x, \tilde{x}$ directions, each wraps a one-cycle within the four directions $u_{1}, u_{2}, \tilde{u}_{1}, \tilde{u}_{2}$. We will consider the generic case that the KK5-branes are not parallel to the D4-brane.

Let us analyze their interaction energy first in the case that they are widely separated. This will produce an $\eta$ problem in the direction of their relative separation, as in [20]. For a large distance $u_{4, K K 5} L_{u} \sqrt{\alpha^{\prime}} \gg \sqrt{\alpha^{\prime}}$ between our D4-brane and the KK5-brane (here not keeping track of the anisotropy $\beta$, which will enter in our more precise analysis below), we 
obtain

$\Delta V_{4, K K 5} \sim \frac{1}{2^{2} \pi^{7 / 2}} \frac{\zeta n_{K}}{L_{u_{2}}} L_{x} V_{\mathcal{R}}(\phi) \log \left(u_{4, K K 5}\right) \sim \frac{\zeta}{2^{2} \pi^{7 / 2}}\left(\frac{M}{K}\right)^{1 / 4} V_{\mathcal{R}}(\phi) \log \left(u_{4, K K 5}\right) \quad$ (far field)

where in the last step we indicated how this contribution scales parametrically with $M$ and $K$, in the solutions [4]. In that setup, the varying string coupling encoded in $\zeta$ is necessary in order to obtain an arbitrarily small ratio $M / K: \zeta \lesssim \mathcal{O}\left((M / K)^{1 / 4}\right)$ in this parametric limit. However, for modest values of the parameters, such as the example studied numerically in [4], and for the minimally tuned model discussed above in \$2.7, we have that this ratio $M / K$ is numerically small.

This contribution (3.70) to the potential, and to the slow-roll parameters in the $\phi$ direction, evidently becomes small in the regime $M \ll K$. However, in this far field configuration, we must check whether significant relative motion between the KK5-brane and the D4-brane is induced by the potential between them. If that happened, the corresponding scalar field $\phi_{4, K K 5}$ would either become the inflaton, or - more generically - would roll too rapidly to produce inflation.

To check this, since $3 H \dot{\phi}=-\partial_{\phi} V$ and $3 H \dot{\phi}_{4, K K 5}=-\partial_{\phi_{4, K K 5}} V$, to ensure that $\dot{\phi}_{4, K K 5} \ll$ $\dot{\phi}$, we must insist that

$$
\left|\partial_{\phi} V\right| \sim \frac{V_{\mathcal{R}}(\phi)}{\phi} \gg\left|\partial_{\phi_{4, K K 5}} V\right| \sim \frac{\zeta}{2^{2} \pi^{7 / 2} \phi_{4, K K 5}}\left(\frac{M}{K}\right)^{1 / 4} V_{\mathcal{R}}(\phi)
$$

which implies

$$
\frac{\zeta}{2^{2} \pi^{7 / 2}}\left(\frac{M}{K}\right)^{1 / 4} \ll \frac{\phi_{4, K K 5}}{\phi}
$$

Canonically normalizing the field $\phi_{4, K K 5}$, we find for the motion mode $\phi_{4, K K 5}$ of the KK5brane

$$
\frac{\phi_{4, K K 5}}{\phi} \sim \frac{M_{\mathrm{P}}}{\phi} \frac{\zeta^{1 / 2}}{(2 \pi)^{7 / 2}}\left(\frac{M}{K}\right)^{1 / 8}
$$

(In this discussion, we are assuming for now - most conservatively - that no other effect masses up this mode.) For the realistic model, we have $\frac{\phi}{M_{\mathrm{P}}} \sim 9$, and the condition (3.72) might be possible to solve, by arranging that $\zeta(K / M)^{1 / 4}$ be somewhat smaller than 1 .

However, in order to avoid a large $\eta$ parameter in the $\phi_{4, K K 5}$ direction, we would need to require - taking two derivatives of $V$ - that

$$
\frac{\zeta}{2^{2} \pi^{7 / 2}}\left(\frac{M}{K}\right)^{1 / 4} \leq\left(\frac{\phi_{4, K K 5}}{\phi}\right)^{2} \quad \text { for } \eta_{\phi_{4, K K 5}} \leq \eta_{\phi}
$$

This, however, does not hold parametrically: the RHS of (3.74) is in fact smaller by a factor of $\left(M_{\mathrm{P}} / \phi\right)^{2} \ll 1$. Therefore without compensating contributions to the mass, we would 
have an $\eta$ problem in this configuration; the interaction energy between the D4-brane and the KK5-branes produces an order 1 contribution to $\eta$. Depending on the precise relative orientation of our ingredients, the curvature, a varying string coupling, and orientifold group actions can contribute to the mass term for $\phi_{4, K K 5}$, and can potentially be used to avoid or cancel against the offending contribution (3.74), as we now sketch.

\subsubsection{A specific setup for string-theoretic $\phi^{2 / 3}$ inflation}

As a specific example (depicted in Figure 3i), wrap the KK5-branes on the cycle generated by the translations $t_{u_{1}} t_{\tilde{u}_{1}} t_{u_{2}}^{1 / b} t_{\tilde{u}_{2}}^{1 / b}$, with $b$ taken to be of order $\beta$ so as to stabilize $L_{2} / L_{1}$ at the value $\beta$. Wrap the D4-brane on the cycle generated by $t_{u_{2}} t_{\tilde{u}_{2}}$, and place it at the point $u_{1}-\tilde{u}_{1}=0$ in the $u_{1}-\tilde{u}_{1}$ direction. It is not mutually BPS with respect to the O6 plane considered in [4]. Our strategy will be to separate this point from the location of the O6plane, and use the local curvature-induced mass obstructing the D4-brane's motion in the $u_{1}-\tilde{u}_{1}$ direction to metastabilize the D4-brane away from the O6-plane and the KK5-brane. (Alternatively, one may try to keep the D4 and KK5 branes together, a scenario we discuss in the context of the $\tilde{\phi}^{2 / 5}$ model in $\$ 3.1 .2$ below.

To this end, take the orientifold action to be the standard worldsheet orientation reversal $\Omega(-1)^{F_{L}}$ combined with the action $u_{2} \rightarrow \tilde{u}_{2}, u_{1} \rightarrow \tilde{u}_{1}+1 / 2, x^{\prime} \rightarrow \tilde{x}^{\prime}-M \tilde{u}_{2} / 2$. This puts the orientifold 6-plane, the fixed point locus of this action, at $u_{1}=\tilde{u}_{1}+1 / 2, u_{2}=\tilde{u}_{2}, x^{\prime}=$ $\tilde{x}^{\prime}-M \tilde{u}_{2} / 2$. On the covering space of the orientifold action, it maps the D4-brane to an anti-D4-brane, so the D4-brane becomes unstable if it gets too close to the O6-plane in the orientifolded space. This is easiest to see perhaps by formally T-dualizing our O6-D4 system to an O2-D0 system. That our D4-brane carries no net charge in the orientifold can also be seen from the fact that its potential field $C_{0123, u_{2}+\tilde{u}_{2}}^{(5)}$ is odd under the orientifold action by virtue of the intrinsic parity under $\Omega(-1)^{F_{L}}$ carried by $C^{(5)}$. Place the KK5-brane at the same position $u_{1}-\tilde{u}_{1}=1 / 2$ as the O6-plane; the orientifold action freezes its motion mode in this direction, projecting out the corresponding transverse motion mode $\phi_{K K 5}$. Inflation corresponds to motion of the D4-brane in the $u_{1}+\tilde{u}_{1}$ direction, i.e. along the direction $u$, where $u_{1}=\tilde{u}_{1} \equiv u$ (and at fixed $u_{2}-\tilde{u}_{2}$ ).

Consider now the curvature-induced potential for D4-brane motion in the $u_{1}-\tilde{u}_{1}$ direction. Setting $u_{1}=u+\Delta u$ and $\tilde{u}_{1}=u-\Delta u$, this contribution to the D4-brane potential energy is of order

$$
V_{D 4, \mathcal{R}}(u, \Delta u) \sim \frac{L_{x} M}{(2 \pi)^{4} g_{s}\left(\alpha^{\prime}\right)^{2}} \sqrt{(u+\Delta u)^{2}+(u-\Delta u)^{2}} \sim V_{\mathcal{R}}(\phi)\left(1+\frac{1}{2} \frac{(\Delta u)^{2}}{u^{2}}+\ldots\right)
$$

where we used the full metric on $\mathcal{N}_{3} \times \mathcal{N}_{3}$, taking into account that the D4-brane is wrapped along the coordinate locus $u_{2}=\tilde{u}_{2}$.

The KK5-brane and the O6-plane also contribute to the potential for $\Delta u$. Note that 


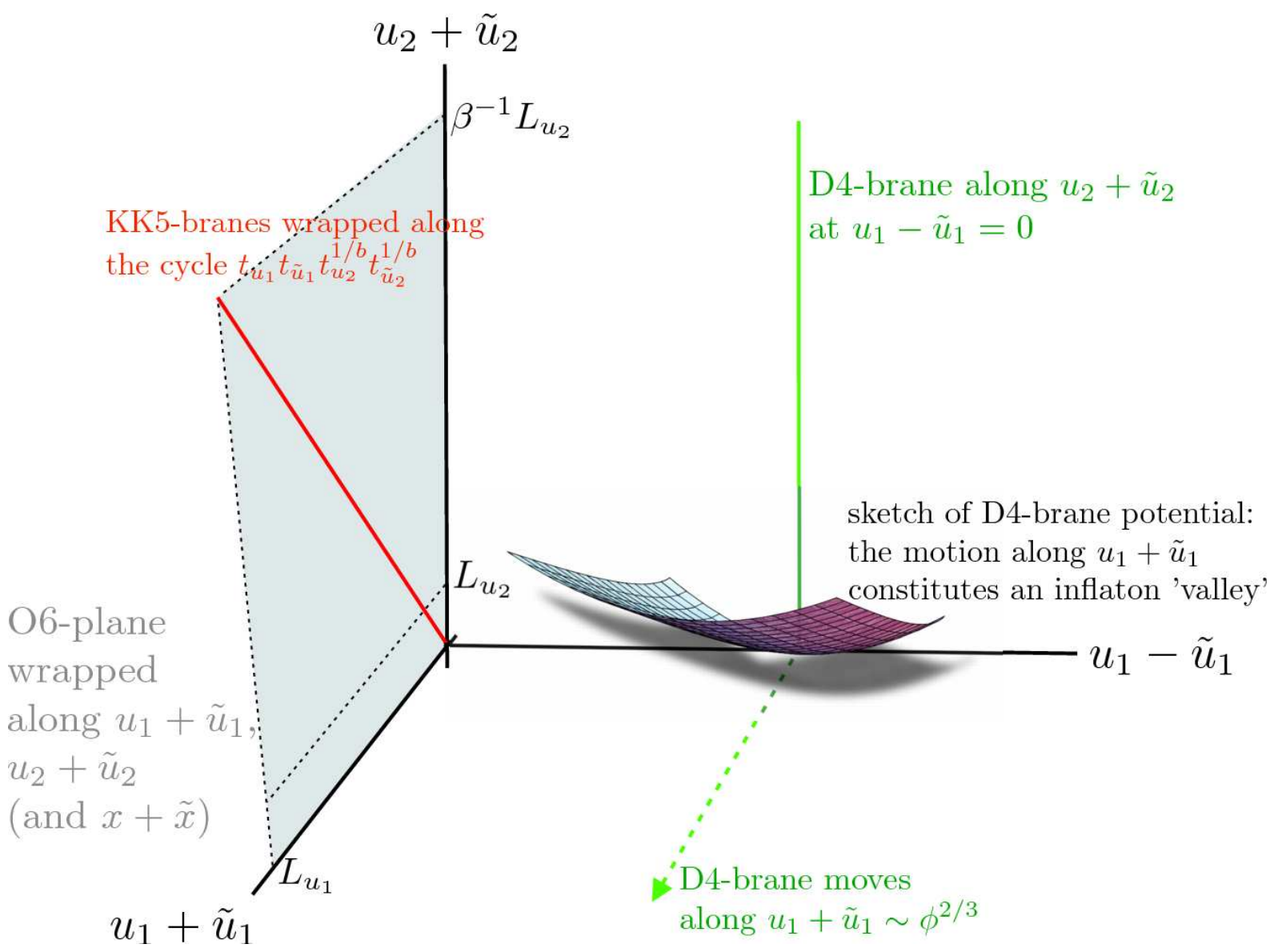

Figure 3; The configuration of moduli-stabilizing ingredients and their relation to our D4brane, in the setup outlined in the text as a method for controlling the slow-roll parameters.

they are arranged symmetrically with respect to the candidate inflaton direction $u$, so do not contribute any $\phi$-dependent corrections. We must check whether the curvature-induced mass just computed in (3.75) suffices to stabilize the D4-brane against motion away from $\Delta u=0$, or whether instead there is a tadpole from the KK5-D4 force which shifts D4 position by a separation $\Delta u$ which is bigger than order 1 .

The D4-O6 force comes in the the open string channel from strings stretching between the D4-brane and its anti-D4-brane partner on the covering space of the orientifold. The KK5-brane, as a leading contribution to the moduli potential, is heavier and should yield the strongest contribution to the potential in the $\Delta u$ direction. We can analyze this starting again from our expression for the interaction energy $\Delta V_{4, K K 5}$ (3.70), now including the appropriate factors of $\beta$ which descend from the KK5 tension and from the fact that we separate the branes in the $u_{1}-\tilde{u}_{1}$ direction (whose proper length scales like $L_{u_{1}}\left(u_{1}-\tilde{u}_{1}\right)$ ). This gives

$$
\Delta V_{4, K K 5} \sim \frac{\beta^{1 / 2}}{2^{2} \pi^{7 / 2}} \frac{\zeta M L_{x}}{L_{2}} V_{\mathcal{R}}\left(\Delta u-\frac{1}{2}(\Delta u)^{2}+\ldots\right)
$$


Thus in order to suppress the shift $\Delta u$, as well as the negative contribution to the mass squared, coming from the D4-KK5 interaction energy, we must require

$$
\frac{\zeta \beta^{1 / 2} M L_{x} u^{2}}{2^{2} \pi^{7 / 2} L_{2}} \lesssim \mathcal{O}(1)
$$

The left hand side is $\lesssim \zeta \beta^{1 / 2} / M^{1 / 2}$ in our specific example discussed in 92.7 , and hence this condition can be satisfied.

We also find that this orientation is locally stable against tilting of the D4-brane. Tilting them increases the length and hence introduces a mass term; this also turns out to dominate over the attractive tadpole between the separated segments of D4 and KK5 (estimated from (3.70) ), leading to only a small tilting of the brane.

\subsubsection{A setup for the version with a $\tilde{\phi}^{2 / 5}$ potential}

Let us also briefly discuss a mechanism for controlling these corrections to $\epsilon$ and $\eta$ in the case of the $\tilde{\phi}^{2 / 5}$ model discussed in $\$ 2.3 .3$. If we wrap the D4-brane in the direction $u_{2}-\tilde{u}_{2}$, then it is mutually BPS with respect to the O6-plane, to leading order. In this case, we can deal with the mutual attraction between the D4-brane and the KK5-brane by placing them together so that they intersect. We can maintain this throughout the evolution if we move the D4-brane appropriately along $u_{2}+\tilde{u}_{2}$ as well as $u_{1}+\tilde{u}_{1}$. Motion in both these directions corresponds to a model with a $\tilde{\phi}^{2 / 5}$ potential as outlined in $\$ 2.3 .3$. In this example, as in the previous one, the O6-plane is also homogeneously extended along the direction of motion of the D4-brane.

In this configuration, however, the D4-brane carries charge since it is mutually BPS with respect to the O6-plane. So let us discuss the effects of the $\overline{\mathrm{D} 4}$-brane charge which must also be included in this version of the construction. We could include an explicit $\overline{\mathrm{D}} 4$-brane, separated from the D4-brane, for example, and try to locally stabilize it or otherwise cancel its contribution to $\eta$ using other effects. However, a cleaner approach is to use fluxes to cancel the brane charge, as in [24]. In our case, the Chern-Simons term $\int C_{5} \wedge F_{2} \wedge H$ yields a source for the RR potential coupling to the D4-brane $\left(C_{5}\right)$ from internal RR and NS fluxes $\int F_{2} \wedge H$.

In order to use this method, we need to ensure that the added RR 2-form flux is compatible with the rest of the ingredients used for moduli stabilization. First, note that as reviewed in [4], the $Z_{2}$ symmetry by which we orientifold to produce the O6-plane acts with a $(-1)$ on $C_{1}$ and $C_{5}$ as well as on $B_{2}$, in addition to its geometrical action exchanging the tilded and untilded coordinates. This is consistent with the coupling we wish to use, given the orientation of our D4-brane along $u_{2}-\tilde{u}_{2}$ : it implies that $C_{5}=C_{5,0123 u_{2-}}\left(d u_{2}-d \tilde{u}_{2}\right) \wedge d x^{0} \wedge d x^{1} \wedge d x^{2} \wedge d x^{3}$, which is altogether invariant under the orientifold action. The $H$ flux in [4] (eqn 3.13) is also of course invariant under the $\mathrm{O} 6$ action, so the introduction of an invariant 2-form RR flux of the form $F_{2}=Q_{2}\left[d u_{1} \wedge\left(d x^{\prime}+M u_{1} d u_{2}\right)-d \tilde{u}_{1} \wedge\left(d \tilde{x}^{\prime}+M \tilde{u}_{1} d \tilde{u}_{2}\right)\right]$ is compatible with the 
symmetry by which we wish to orientifold. We must also check that the potential energy $\mathcal{U}_{F_{2}}$ introduced by $F_{2}$ is compatible with moduli stabilization. This holds as well:

$$
\mathcal{U}_{F_{2}} \sim M_{\mathrm{P}}^{4} \frac{g_{s}^{4}}{L^{6}} \frac{Q_{2}^{2}}{L_{x}^{2} L_{u_{1}}^{2}} \sim \beta\left(\frac{M}{K}\right)^{1 / 2} Q_{2}^{2} \mathcal{U}_{\text {mod, }, \mathcal{R}} \ll \mathcal{U}_{\text {mod, } \mathcal{R}}
$$

\subsubsection{General comments on the $\eta$ problem}

As emphasized above, in order to produce a reliable model of large-field inflation, our control over $\epsilon$ and $\eta$ must hold over the entire super-Planckian range of $\phi$. In the present top-down construction, we use the methods just described to control the dynamics of the D4-brane in each $\Delta\left(u_{1}+\tilde{u}_{1}\right)=1$ interval. As the D4-brane moves around the $u_{1}+\tilde{u}_{1}$ direction multiple times, its potential energy increases as it wraps a longer cycle on the $T^{2}$ traced out by $x^{\prime}+\tilde{x}^{\prime}$ and $u_{2}+\tilde{u}_{2}$. But the basic methods we outlined to control its potential apply in each such interval. Thus it seems that roughly speaking, the problem of controlling inflation in this setup - even large-field inflation - reduces to the problem of computing and suppressing contributions to $\epsilon$ and $\eta$ for brane inflation [20] in a sub-Planckian range of field space.

Even given this, the problem of controlling inflation in each sub-Planckian range of field space is itself extremely subtle, as explained in the series of works [20, 21, 22, 23]. For example in brane inflation, the interaction energy between the D-brane and other branes or localized moduli stabilizing ingredients get significant $\phi$ and volume-dependent contributions from fields propagating around the compact manifold (as well as from fields propagating directly between the defects) [23, 20]. This is true as well in our case, but with the specifications we have made the symmetries cancel some of these contributions against each other: the D4-brane's collective coordinate $u_{1}+\tilde{u}_{1}$ does not correspond to the distance between the brane and other localized sources of stress-energy at leading order 11

We should reiterate another set of subtleties in this construction. We have been considering the simplest version of the construction [4], which leaves a degeneracy between the heaviest moduli and lightest KK modes as discussed there. Additional ingredients (such as NS5-branes) introduced to split these scales would entail further potential contributions to the candidate inflaton trajectory. As discussed in [4], the angular moduli are also rather subtle, but the moduli potential does seem to exhibit local minima in these directions whose precise location we have not computed. In any case, the mechanism we propose in the present work would apply much more generally in compactifications in which branes undergo monodromies, assuming they can be stabilized with ingredients manifesting the requisite symmetries.

All existing constructions have some subtleties of this sort, and it is fair to say that small-field models seem a priori easier to implement via a tuned cancellation of corrections

\footnotetext{
${ }^{11}$ See e.g. [28] for a different use of symmetries, within a small-field inflationary scenario, to cancel contributions to the slow-roll parameters.
} 
to the inflaton mass within a small range of $\phi$. In some ways, however, the present case is somewhat more straightforward to analyze than, say, a warped Calabi-Yau manifold, as the compactification manifold $\mathcal{N}_{3} \times \tilde{\mathcal{N}}_{3}$ itself is extremely simple. Of course more generally, manifolds with metric flux are more generic and perhaps typically more complicated than Calabi-Yau manifolds. It would be very interesting to understand the range of possible behaviors of the potential in a wider class of manifolds with metric and generalized fluxes.

\subsubsection{The Standard Model}

We should emphasize that our construction does not yet include an explicit Standard Model sector. This is of course necessary for a fully realistic model, and may be challenging since each additional defect may lead to a new source of large corrections to the slow-roll parameters. One may approach this by considering e.g. a "brane box" construction, with D4-branes suspended between 5-branes, or an intersecting D6-brane construction. In each case, the effects on moduli stabilization and on the inflaton potential would need to be analyzed and controlled. We leave this for future work. Including the Standard Model is also a prerequisite for a detailed study of reheating (see [33] for a recent analysis of this issue in N-flation).

\section{$3.2 \quad \alpha^{\prime}$ and Loop Corrections}

Let us next estimate the $\alpha^{\prime}$ and loop corrections to $\epsilon$ and $\eta$. The $\alpha^{\prime}$ corrections arise from corrections to $\Phi(X), G_{M N}(X)$, and fluxes to which the D4-brane couples through the effective action (2.4), as well as corrections to the form of this action from higher derivatives of the

bulk fields. To get a sense of the issues, us start with corrections to the potential from $\alpha^{\prime}$ effects $\left(V \rightarrow V+\Delta_{\alpha^{\prime}} V\right)$ which are schematically of the form

$$
\Delta_{\alpha^{\prime}} V \sim V_{\mathcal{R}} \sum_{n} c_{n}\left(\mathcal{R} \alpha^{\prime}\right)^{n}
$$

(where " $\left(\mathcal{R} \alpha^{\prime}\right)^{n}$ refers to scalars made from appropriate contractions of components of the Riemann and metric tensors) plus similar terms in which the curvature is replaced by fluxes or dilaton gradients, where $c_{n}$ are order one constants. In our setting of $10 \mathrm{~d}$ type II string theory, the $n=4$ term is the leading $\alpha^{\prime}$ correction to the bulk metric. Each term in (3.79) gives a correction to $\epsilon$ and $\eta$ of order

$$
\Delta_{\alpha^{\prime}} \epsilon \sim \frac{\partial}{\partial\left(\phi / M_{\mathrm{P}}\right)}\left(\mathcal{R} \alpha^{\prime}\right)^{n}, \quad \Delta_{\alpha^{\prime}} \eta \sim \epsilon \Delta+\frac{\partial^{2}}{\partial\left(\phi / M_{\mathrm{P}}\right)^{2}}\left(\mathcal{R} \alpha^{\prime}\right)^{n}
$$

Since the curvatures (and other field strengths) and their derivatives are small in our background, we find that the effect of the $\alpha^{\prime}$ corrections on the slow variation parameters is small. In our regime $\phi \gg \phi_{\text {crit }} \Rightarrow L_{x} M u_{1} \gg L_{u_{2}}$, all components of the metric and Riemann tensors of the Nil manifolds are proportional to an order 1 power of $\phi$. (They all depend 
on $u_{1}$, if at all, through a combination of the form $c_{1} L_{u_{2}}^{2}+L_{x}^{2} M^{2} u_{1}^{2}$ where $c_{1}$ is of order 1.) Therefore their derivatives are at most of order

$$
M_{\mathrm{P}}^{n} \partial_{\phi}^{n}\left(\mathcal{R} \alpha^{\prime}\right)^{n^{\prime}} \lesssim\left(\frac{M_{\mathrm{P}}}{\phi}\right)^{n}\left(\mathcal{R} \alpha^{\prime}\right)^{n^{\prime}}
$$

Similarly,

$$
M_{\mathrm{P}}^{n} \partial_{\phi}^{n} V_{\mathcal{R}} \sim\left(\frac{M_{\mathrm{P}}}{\phi}\right)^{n} V_{\mathcal{R}}
$$

so since $\frac{M_{\mathrm{P}}}{\phi} \sim 10^{-1}$ and the curvatures $\mathcal{R} \alpha^{\prime}$ are small, the corrections to $\epsilon$ and $\eta$ from terms of the form (3.79) are negligible in our background.

More precisely, we recall the derivation of the DBI-form of the D-brane action from the calculation of the beta functions of the boundary action in the worldsheet sigma model [29]. It was shown there by the structure of worldsheet 1-loop diagrams determining the beta function of the coupling corresponding to the world volume gauge field $A_{M}$ that the DBIform of the D-brane action holds for all orders in $\alpha^{\prime}$ up to first derivatives of $\Phi, B_{M N} \& F_{M N}$ and second derivatives in $G_{M N}$ and through 1-loop in the string coupling. To this order therefore all corrections thus appear through the corrected $G_{M N}, B_{M N}$, and $\Phi$ alone which has already been taken into account. Let us now analyze the first curvature corrections which can appear in this action at the level of second derivatives of $G_{M N}$.

Schematically, the leading correction at $\mathcal{O}\left(\alpha^{\prime}\right)$ in the curvature to the D4-brane action is by the symmetries of the action

$$
S_{\mathrm{D} 4} \sim \int d^{5} \xi \sqrt{\operatorname{det}\left(G_{M N}+\alpha^{\prime} c_{\mathcal{R}} \mathcal{R}_{M N}\right) \partial_{\alpha} X^{M} \partial_{\beta} X^{N}}\left(1+\tilde{c}_{\mathcal{R}} \alpha^{\prime} \mathcal{R}+\ldots\right)
$$

where $c_{\mathcal{R}}, \tilde{c}_{\mathcal{R}}=\mathcal{O}(1)$. The Ricci scalar $\mathcal{R}$ is small and independent of position inside the manifold. The relevant nonzero components of the Ricci tensor are (for simplicity suppressing the $\beta$ dependence)

$$
\alpha^{\prime} R_{u_{1} u_{1}}=-\frac{L_{x}^{2} M^{2}}{2 L_{u}^{2}} \quad \alpha^{\prime} R_{u_{2} u_{2}}=-\frac{L_{x}^{2} M^{2}}{2 L_{u}^{4}}\left(L_{u}^{2}-L_{x}^{2} M^{2} u_{1}^{2}\right)
$$

Comparing these to the metric components $G_{u_{1} u_{1}}$ and $G_{u_{2} u_{2}}$ we see they do not correct the shape of the potential and are highly suppressed. (Higher powers of curvature could change the shape of the potential at some order, but are further suppressed.)

Next let us address loop corrections to our background. The center of mass position of our D4-brane is governed by an abelian worldvolume theory. In a flat spacetime background, the DBI action for the probe does not receive loop corrections, and the absence of a potential term is guaranteed in that case by translation invariance. In our setup, the only corrections to the potential therefore arise by virtue of the ambient curvature, and are suppressed as in the previous discussion (as well as by factors of the weak string coupling). Nonetheless it 
is interesting to consider the structure of loop corrections in the four-dimensional effective theory.

The quantum effective potential will have some dependence on the position of our D4brane, and hence on $\phi$. As a specific example of a 1-loop effect which manifests a $\phi$-dependent correction to the $\phi^{2 / 3}$ form of our potential, consider the self-interactions of the scalar field perturbations $\delta \phi$ implied by our leading potential $V_{\mathcal{R}} \sim \mu^{10 / 3} \phi^{2 / 3} \equiv \mu^{10 / 3}\left(\phi_{0}+\delta \phi\right.$ ) (where $\phi_{0}=\phi_{0}(t)$ denotes the background evolution of the inflaton). We have

$$
V_{\mathcal{R}} \sim \cdots+\lambda_{3} \delta \phi^{3}+\lambda_{4} \delta \phi^{4}+\ldots
$$

with $\lambda_{3} \sim \mu^{10 / 3} / \phi_{0}^{7 / 3} \sim 10^{-12} M_{\mathrm{P}}$ and $\lambda_{4} \sim\left(\mu / \phi_{0}\right)^{10 / 3} \sim 10^{-13}$. These terms generate, via 1loop diagrams, corrections to the inflaton mass squared of order $\Delta m_{\phi}^{2} \sim \frac{1}{(2 \pi)^{4}}\left(\lambda_{3}^{2}+M_{S U S Y}^{2} \lambda_{4}\right)$, where $M_{S U S Y}$ is the SUSY breaking scale which cuts off the loop in the second case. The inflaton mass squared in our original potential, $m_{\phi}^{2} \sim \partial_{\phi}^{2} V_{\mathcal{R}}$, is of order $10^{-4} H^{2} \sim 10^{-12} M_{\mathrm{P}}^{2}$, much greater than these loop corrections.

More generally, consider the structure of the 1-loop vacuum amplitude. It depends on $\phi$ through the dependence on the brane position $u_{1}$ of the masses of KK and string modes which propagate in the loop. Schematically, the partition function is of the form

$$
\Delta V_{1-\text { loop }} \sim \frac{1}{\left(\alpha^{\prime}\right)^{2}} \int d^{4} k \int \frac{d \tau_{2}}{\tau_{2}}\left(\sum_{m_{B}, n_{B}} e^{-\left(m_{B}^{2}+p_{n_{B}}^{2}+k^{2}\right) \alpha^{\prime} \tau_{2}}-\sum_{m_{F}, n_{F}} e^{-\left(m_{F}^{2}+p_{n_{F}}^{2}+k^{2}\right) \alpha^{\prime} \tau_{2}}\right)
$$

where $k$ is the four-dimensional momentum. Here $m_{B}$ and $m_{F}$ are the boson and fermion string masses and $p_{n_{B}}^{2}$ and $p_{n_{F}}^{2}$ are the boson and fermion KK mode mass squareds. Integrating over $k$ and expanding this out, we obtain as a conservative estimate for the size of this loop correction

$$
\Delta V_{1-\text { loop }} \leq \frac{1}{\left(\alpha^{\prime}\right)^{2}}\left(M_{S U S Y}^{2} \alpha^{\prime}\right) \sim M_{\mathrm{P}}^{4} \frac{(2 \pi)^{14}}{4} g^{4}\left(M_{S U S Y}^{2} \alpha^{\prime}\right) \sim g\left(M_{S U S Y}^{2} \alpha^{\prime}\right) \mathcal{U}_{\text {mod }, \mathcal{R}}
$$

where $M_{S U S Y}$ is again the effective SUSY breaking scale which cuts off the loop, and where we used the fact that all the leading terms in the moduli potential in 4, such as the orientifold term $\sim-g^{3} M_{P}^{4}$, scale like $\mathcal{U}_{m o d, \mathcal{R}}$ in the solution. The leading effects which break both supersymmetry and translation invariance in the $u_{1}$ direction come from the Nil manifold curvature, so we have that $M_{S U S Y}^{2} \alpha^{\prime}$ is of the form

$$
M_{S U S Y}^{2} \alpha^{\prime} \sim \sum_{n} c_{n}\left(\mathcal{R} \alpha^{\prime}\right)^{n}
$$

Now since $g\left(M_{S U S Y}^{2} \alpha^{\prime}\right) \mathcal{U}_{\text {mod, } \mathcal{R}} \ll \mathcal{U}_{\text {mod, } \mathcal{R}}$, there is a large window in which $g\left(M_{S U S Y}^{2} \alpha^{\prime}\right) \mathcal{U}_{\text {mod, } \mathcal{R}} \ll$ $V_{\mathcal{R}} \leq \mathcal{U}_{\text {mod, } \mathcal{R}}$. Combining this with the fact discussed above that derivatives with respect to $\phi / M_{\mathrm{P}}$ pull down inverse powers of $\phi / M_{\mathrm{P}}$, we see that $M_{\mathrm{P}}^{n} \partial_{\phi}^{n} \Delta V_{1-\text { loop }} \ll M_{\mathrm{P}}^{n} \partial_{\phi}^{n} V_{\mathcal{R}}$, which implies that the corrections to $\epsilon$ and $\eta$ from the 1-loop effective potential are small. 
Let us next check the renormalization of Newton's constant as in [12], given by

$$
M_{\mathrm{P}, \text { renorm }}^{2}=M_{\mathrm{P}}^{2}+N_{s} \Lambda^{2}
$$

where $N_{s}$ is the number of species and where $\Lambda$ is the effective UV cutoff in loop momentum.. In discussing the absence of back reaction of the D4-brane on the geometry in \$2.4.3, we found the effective number of species added by our mechanism (2.47). In the specific example discussed above in $\$ 2.7$, this was of order 20 , or of order $10^{2}$ if we count the relatively heavy open strings between the multiple wrappings of the brane. Even if $\Lambda$ were as high as string scale, the correction is negligible since $\frac{1}{\alpha^{\prime}} \sim \frac{(2 \pi)^{7}}{2} g^{2} M_{\mathrm{P}}^{2} \sim \frac{(2 \pi)^{7}}{2} \frac{\hat{g}^{2}}{K^{2}} M_{\mathrm{P}}^{2} \sim 7 \times 10^{-5} M_{\mathrm{P}}^{2}$.

\section{Observational Predictions}

We shall thus now derive the observational predictions from this inflationary regime. For the minimal case of a single-field slow-roll model, our theory predicts negligible non-Gaussianity. As such, it is cleanly distinguishable from single-field models with low sound speed, and from a large class of multifield models. (However, it is possible that the same mechanism could be generalized to cases with light transverse fields to match an observed $f_{N L}^{\text {local }}$ if present.)

The remaining CMB observables are the spectral index $n_{s}$ of the curvature perturbation and the tensor-to-scalar ratio $r$ of primordial gravitational waves generated during inflation. In the slow-roll approximation these quantities at $N$ efolds before the end of inflation are given by

$$
\begin{aligned}
\left.n_{s}\right|_{N} & =1-6 \epsilon_{N}+2 \eta_{N} \\
\left.r\right|_{N} & =16 \epsilon_{N}
\end{aligned}
$$

where the index $N$ indicates that these quantities are to be calculated at about $N$ efolds before the end of inflation, i.e. at $\phi_{N}$.

Here, we can determine $n_{s}$ and $r$ in general only numerically as $V_{\mathcal{R}}(\phi)$ is given in its full form only numerically. However, in the limiting case of a pure power law potential $V(\phi) \propto \phi^{p}$, these observables are given by

$$
\begin{aligned}
\left.n_{s}\right|_{N} & =1-\frac{2+p}{2 N} \\
\left.r\right|_{N} & =\frac{4 p}{N} .
\end{aligned}
$$

At about 60 efolds before the end of inflation, when the COBE normalization scale left the horizon, this yields for $m^{2} \phi^{2}$ inflation $n_{s}=0.967$ and $r=0.13$. For our case of the $\mu^{10 / 3} \phi^{2 / 3}$-limit of $V_{\mathcal{R}}(\phi)$ we get

$$
n_{s} \simeq 0.978 \text { and } \quad r \simeq 0.04
$$




\section{Chaotic Inflation}

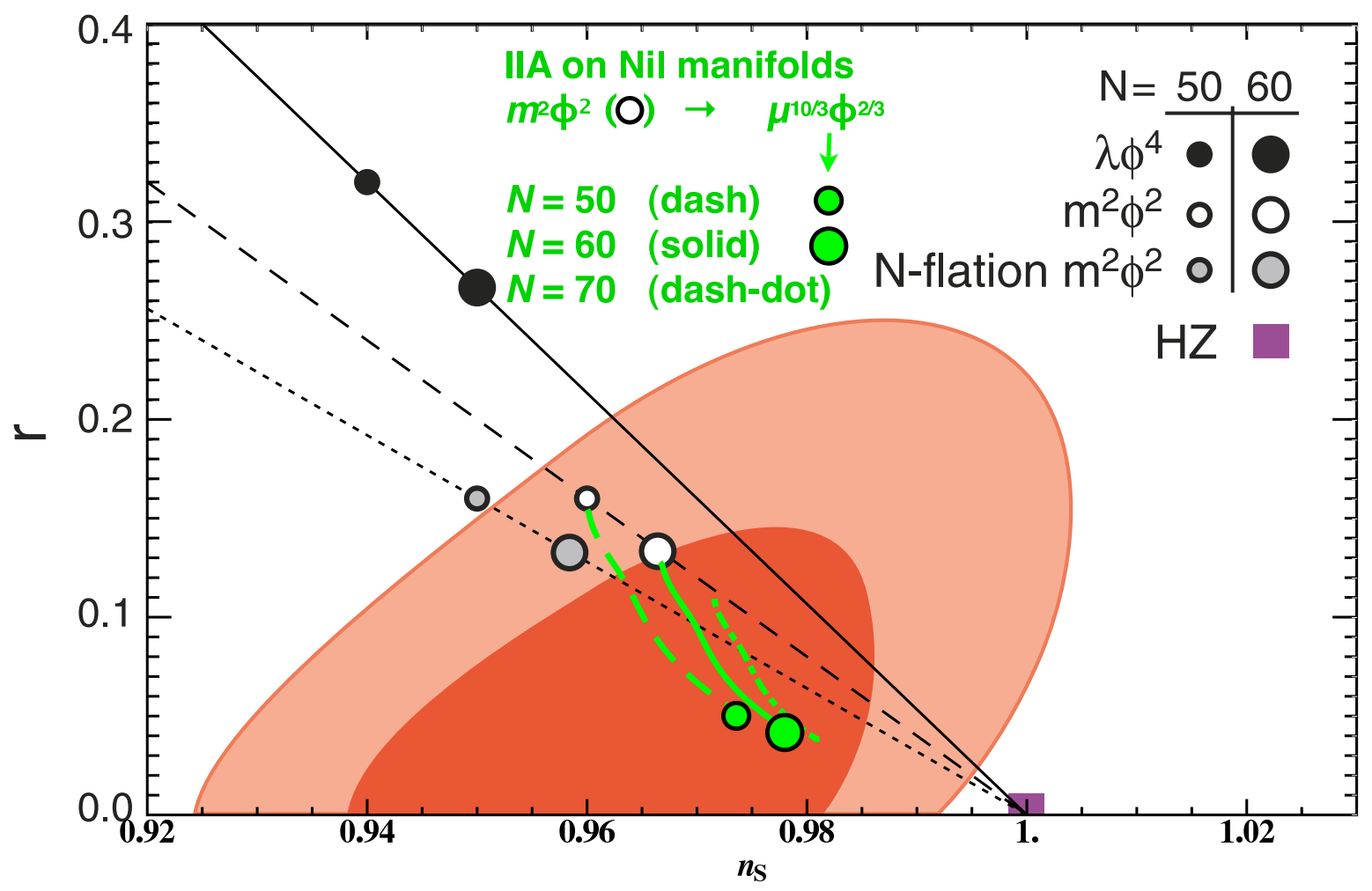

Figure 4: Red: 5-year WMAP+BAO+SN [9] combined joint $68 \%$ and $95 \%$ error contours on $\left(n_{s}, r\right)$. Green: General prediction of the potential $V_{\mathcal{R}}(\phi)(2.14)$ as one formally varies $\beta$ to interpolate between $m^{2} \phi^{2}$ (black hollow circles) and $\mu^{10 / 3} \phi^{2 / 3}$ (green solid circles). Only the latter regime is viable in our setup as discussed in the text, so the solid green circles (for $N=50,60$ efolds before the end of inflation) denote our prediction.

plus in general corrections which are at most of order 0.01 from e.g. the shifts in the moduli induced by the inflaton potential (2.46).

Before taking into account moduli stabilization, the value of $\beta$ formally determines in which of these regimes of the full potential the last 60 efolds of inflation fall, so we can numerically derive $r$ as a function of $n_{s}$ parametrized by their dependence on $\beta$. This prediction is shown by the green curve in Fig. 4 together with the $68 \%$ and $95 \%$ joint error contours of the 5-year WMAP data [9] in the $\left(n_{s}, r\right)$-plane.

We see that the $m^{2} \phi^{2}$-endpoint (the upper left end of the green curve) matches with the open circle denoting the pure $m^{2} \phi^{2}$-potential. Of course, the $m^{2} \phi^{2}$-regime in the string construction here is not viable for the reason explained above that it destabilizes the moduli if we require the necessary 60 efolds of slow-roll inflation. Thus, the observationally viable part of the green line consists of its lower right part whose endpoint (the solid green circle) is to good approximation the pure $\mu^{10 / 3} \phi^{2 / 3}$-potential. 
For the $\tilde{\mu}^{18 / 5} \tilde{\phi}^{2 / 5}$-case discussed above in $\$ 2.3 .3$ and $\$ 3.1 .2$, we note that $n_{s} \simeq 0.98$ and $r \simeq 0.03$.

\section{Discussion}

Let us summarize what we have obtained. By using a monodromy of wrapped branes on Nil manifolds - the fact that their approximate moduli space lives on the covering space of the compact twisted torus - we extended the kinematical field range of brane inflation in a simple way. The resulting candidate inflaton has an asymptotically power-law potential determined by the compactification geometry. This potential is proportional to $\phi^{2 / 3}$ in the case studied in detail, and we also find a variant with a $\tilde{\phi}^{2 / 5}$ potential corresponding to a different direction of motion of the brane.

We analyzed a host of dynamical and observational conditions for a viable model. We imposed the condition that the inflaton potential $V_{\mathcal{R}}(\phi)$ be subdominant to the modulistabilizing potential and cause negligible back reaction in the geometry, and that the small $\phi$-dependent shifts in moduli not destabilize the inflaton trajectory. These dynamical requirements proved to be consistent with motion over a super-Planckian range of field space. We argued that by orienting our D4-brane in a symmetric manner with respect to the basic moduli-stabilizing ingredients, we can avoid order 1 corrections to the slow-roll parameters $\epsilon$ and $\eta$. In analyzing this, we noted that the monodromy also provides some simplification of the problem that one would a priori expect to need a functional fine-tune of the potential: in our mechanism this large range in field space is built up out of a set of similar, shorter segments corresponding to the motion of the brane once around the compact manifold.

Putting these conditions together, we found that introducing a few percent fine-tune (encoded in our parameter $\beta$ and the flux quantum number $f_{6}$ ), we obtain a viable model realizing this mechanism. The resulting predictions for the tilt and tensor spectrum sit comfortably within the 1-sigma contours obtained from present data, and are testable in upcoming experiments.

As explained in for example [32], there is an interesting tension between the observation of gravity waves and the scale of the moduli potential barrier (which can in some cases be related to the scale of supersymmetry breaking). Here we find that compactification manifolds with a larger potential barrier coming from negative scalar curvature provide a reasonably natural mechanism for high-scale inflation.

We note again that our construction so far contains no Standard Model sector, whose inclusion would yield new challenges for controlling the slow-roll parameters. Moreover our underlying de Sitter compactification, like all such constructions, has a number of subtleties (described in [4]). In particular, the off-diagonal elements in the mass matrix mixing $g, L, L_{x}$ with the angular moduli and with the lightest KK modes has not been explicitly calculated, 
and depend on order 1 coefficients. There appear to be sufficient ingredients available to tune these entries if necessary, but this has not been carried out explicitly. We leave a more detailed analysis of these issues for future work. Similar open questions arise for all string inflationary models, and it would be interesting to work towards a fully explicit construction of inflation combined with moduli stabilization.

Monodromies of the sort used here arise in a broad class of string compactifications, including more general twisted tori and also non-geometrical spaces [10, 18], as well as in motion on the closed string moduli space in Calabi-Yau compactifications [34]. It will be interesting to characterize the potentials that arise in viable models much more generally, in the special cases where the inflaton direction is sufficiently homogeneous to allow control over $\epsilon$ and $\eta$.

Let us also note that we chose to include a single wrapped D4-brane in the compactification, with potential minimized at $u_{1}=0$, but another mechanism for inflation - "trapped inflation" [35] - suggests itself in this geometry. Since the $T^{2}$ traced out by $x^{\prime}, u_{2}$ is equivalent at each position $u_{1}=j / M$ (for integer $j$ ), there is a place for a locally stabilized wrapped D4-brane at each of these places. If present, such extra D4-branes introduce points with extra light species along the inflaton trajectory. The inflaton dumps some energy into these species, since they get produced when it hits these points. This motivates a careful study of the conditions for and predictions of trapped inflation [36].

In general, one may wish to assess how contrived a given construction of observationally testable inflation looks from a model-building point of view. Indeed, without considering specific mechanisms, the problem of testing UV-sensitive contributions to inflation is very difficult a priori [37]. The present construction involves modest fine-tuning and a simple mechanism for enhancing the geometric field range (which should arise in a wide class of compactifications), but requires symmetries used to suppress destabilizing corrections to the slow-roll parameters (which entails special choices, as with all symmetry principles). It is perhaps worth noting that this work began not as a direct attempt to engineer a gravitational wave signature from string inflation, but simply as an investigation of the prospects for inflation of any kind in the setup [4]; the present mechanism and [36] are what emerged thus far from this study. Similar comments apply to other corners of field theory and string theory with predictive inflationary signatures such as [27, 38, 39, 40]. The development of a detailed understanding of UV complete cosmological solutions is just beginning, and it is not yet clear how to put a measure on the space of inflationary mechanisms. However, it is already apparent that upcoming data will be very effective in deciding among wildly different possibilities for field- and string-theoretic inflationary mechanisms. 


\section{Acknowledgments}

We dedicate this work to A. Linde on the occasion of his 60th birthday celebration. We thank T. Banks, J.R. Bond, G. Efstathiou, S. Kachru, R. Kallosh, C.-L. Kuo, A. Lawrence, A. Linde, J. Maldacena, L. McAllister, B. Netterfield, L. Senatore, and D. Tong for useful discussions. The research of E.S. is supported by NSF grant PHY-0244728, by the DOE under contract DE-AC03-76SF00515, and by BSF and FQXi grants. The research of A.W. is supported in part by the Alexander-von-Humboldt foundation, as well as by NSF grant PHY-0244728.

\section{References}

[1] A. H. Guth, "The Inflationary Universe: A Possible Solution To The Horizon And Flatness Problems," Phys. Rev. D 23, 347 (1981);

A. D. Linde, "A New Inflationary Universe Scenario: A Possible Solution Of The Horizon, Flatness, Homogeneity, Isotropy And Primordial Monopole Problems," Phys. Lett. B 108, 389 (1982);

A. Albrecht and P. J. Steinhardt, "Cosmology For Grand Unified Theories With Radiatively Induced Symmetry Breaking," Phys. Rev. Lett. 48, 1220 (1982).

[2] C. P. Burgess, P. Martineau, F. Quevedo, G. Rajesh and R. J. Zhang, "Brane antibrane inflation in orbifold and orientifold models," JHEP 0203, 052 (2002) arXiv:hep-th/0111025.

S. H. Henry Tye, "Brane inflation: String theory viewed from the cosmos," arXiv:hep-th/0610221.

J. M. Cline, "String cosmology," arXiv:hep-th/0612129.

R. Kallosh, "On Inflation in String Theory," arXiv:hep-th/0702059.

C. P. Burgess, "Lectures on Cosmic Inflation and its Potential Stringy Realizations," arXiv:0708.2865 [hep-th].

L. McAllister and E. Silverstein, "String Cosmology: A Review," Gen. Rel. Grav. 40, 565 (2008) [arXiv:0710.2951 [hep-th]].

[3] J. Scherk and J. H. Schwarz, "How To Get Masses From Extra Dimensions," Nucl. Phys. B 153, 61 (1979);

N. Kaloper and R. C. Myers, "The O(dd) story of massive supergravity," JHEP 9905, 010 (1999) arXiv:hep-th/9901045.

S. Kachru, M. B. Schulz, P. K. Tripathy and S. P. Trivedi, "New supersymmetric string compactifications," JHEP 0303, 061 (2003) [arXiv:hep-th/0211182.

C. M. Hull and R. A. Reid-Edwards, "Flux compactifications of string theory on twisted tori," arXiv:hep-th/0503114. 
[4] E. Silverstein, "Simple de Sitter Solutions," to appear in Phys. Rev. D, arXiv:0712.1196 [hep-th].

[5] A. D. Linde, "Chaotic Inflation," Phys. Lett. B 129, 177 (1983).

[6] D. H. Lyth, "What would we learn by detecting a gravitational wave signal in the cosmic microwave background anisotropy?," Phys. Rev. Lett. 78, 1861 (1997) arXiv:hep-ph/9606387.

G. Efstathiou and K. J. Mack, "The Lyth Bound Revisited," JCAP 0505, 008 (2005) arXiv:astro-ph/0503360.

[7] M. Zaldarriaga and U. Seljak, "An All-Sky Analysis of Polarization in the Microwave Background," Phys. Rev. D 55, 1830 (1997) arXiv:astro-ph/9609170.

M. Kamionkowski, A. Kosowsky and A. Stebbins, "Statistics of Cosmic Microwave Background Polarization," Phys. Rev. D 55, 7368 (1997) arXiv:astro-ph/9611125.

G. Efstathiou, "The Future of Cosmology," arXiv:0712.1513 [astro-ph].

G. Efstathiou and S. Chongchitnan, "The search for primordial tensor modes," Prog. Theor. Phys. Suppl. 163, 204 (2006) arXiv:astro-ph/0603118.

[8] A. C. Taylor [the Clover Collaboration], "Clover - A B-mode polarization experiment," New Astron. Rev. 50, 993 (2006) arXiv:astro-ph/0610716.

C. J. MacTavish et al., "Spider Optimization: Probing the Systematics of a Large Scale B-Mode Experiment," arXiv:0710.0375 [astro-ph].

K. W. Yoon et al., "The Robinson Gravitational Wave Background Telescope (BICEP): a bolometric large angular scale CMB polarimeter," arXiv:astro-ph/0606278.

D. Samtleben for the QUIET Collaboration, "Measuring the Cosmic Microwave Background Radiation (CMBR) polarization with QUIET," arXiv:0802.2657 [astro-ph].

J. Bock et al., "Task Force on Cosmic Microwave Background Research," arXiv:astro-ph/0604101.

[9] D. N. Spergel et al. [WMAP Collaboration], "First Year Wilkinson Microwave Anisotropy Probe (WMAP) Observations: Determination of Cosmological Parameters," Astrophys. J. Suppl. 148, 175 (2003) arXiv:astro-ph/0302209;

H. V. Peiris et al., "First year Wilkinson Microwave Anisotropy Probe (WMAP) observations: Implications for inflation," Astrophys. J. Suppl. 148, 213 (2003) arXiv:astro-ph/0302225;

D. N. Spergel et al. [WMAP Collaboration], "Wilkinson Microwave Anisotropy Probe (WMAP) three year results: Implications for cosmology," Astrophys. J. Suppl. 170, 377 (2007) arXiv:astro-ph/0603449. 
E. Komatsu et al., "Five-Year Wilkinson Microwave Anisotropy Probe (WMAP) Observations: Cosmological Interpretation," submitted to Astrophys. J. Suppl. arXiv:0803.0547 [astro-ph].

F. R. Bouchet [Planck Collaboration], "The Planck satellite: Status \& perspectives," Mod. Phys. Lett. A 22, 1857 (2007).

[10] C. M. Hull, "A geometry for non-geometric string backgrounds," JHEP 0510, 065 (2005) arXiv:hep-th/0406102.

A. Lawrence, M. B. Schulz and B. Wecht, "D-branes in nongeometric backgrounds," JHEP 0607, 038 (2006) [arXiv:hep-th/0602025].

[11] D. Baumann and L. McAllister, "A microscopic limit on gravitational waves from D-brane inflation," arXiv:hep-th/0610285.

[12] S. Dimopoulos, S. Kachru, J. McGreevy and J. G. Wacker, "N-flation," arXiv:hep-th/0507205.

R. Easther and L. McAllister, "Random matrices and the spectrum of N-flation," JCAP 0605, 018 (2006) arXiv:hep-th/0512102].

R. Kallosh, N. Sivanandam and M. Soroush, "Axion Inflation and Gravity Waves in String Theory," Phys. Rev. D 77, 043501 (2008) [arXiv:0710.3429 [hep-th]].

T. W. Grimm, "Axion Inflation in Type II String Theory," arXiv:0710.3883 [hep-th].

J. E. Kim, H. P. Nilles and M. Peloso, "Completing natural inflation," JCAP 0501, 005 (2005) arXiv:hep-ph/0409138.

[13] J. R. Bond, L. Kofman, S. Prokushkin and P. M. Vaudrevange, "Roulette inflation with Kaehler moduli and their axions," Phys. Rev. D 75, 123511 (2007) arXiv:hep-th/0612197.

[14] J. J. Blanco-Pillado et al., "Racetrack inflation," JHEP 0411, 063 (2004) arXiv:hep-th/0406230.

[15] J. E. Lidsey and I. Huston, "Gravitational wave constraints on Dirac-Born-Infeld inflation," JCAP 0707, 002 (2007) [arXiv:0705.0240 [hep-th]].

M. Becker, L. Leblond and S. E. Shandera, "Inflation from Wrapped Branes," arXiv:0709.1170 [hep-th];

T. Kobayashi, S. Mukohyama and S. Kinoshita, "Constraints on Wrapped DBI Inflation in a Warped Throat," arXiv:0708.4285 [hep-th].

A. Krause, "Large Gravitational Waves and Lyth Bound in Multi Brane Inflation," arXiv:0708.4414 [hep-th].

I. Huston, J. E. Lidsey, S. Thomas and J. Ward, "Gravitational Wave Constraints on Multi-Brane Inflation," arXiv:0802.0398 [hep-th]. 
[16] A. R. Frey, "Warped strings: Self-dual flux and contemporary compactifications," arXiv:hep-th/0308156.

E. Silverstein, "TASI / PiTP / ISS lectures on moduli and microphysics," arXiv:hep-th/0405068.

M. Grana, "Flux compactifications in string theory: A comprehensive review," Phys. Rept. 423, 91 (2006) [arXiv:hep-th/0509003].

J. Polchinski, "The cosmological constant and the string landscape," arXiv:hep-th/0603249.

M. R. Douglas and S. Kachru, "Flux compactification," arXiv:hep-th/0610102.

F. Denef, M. R. Douglas and S. Kachru, "Physics of string flux compactifications," arXiv:hep-th/0701050.

R. Bousso, "TASI Lectures on the Cosmological Constant," arXiv:0708.4231 [hep-th].

[17] M. P. Hertzberg, S. Kachru, W. Taylor and M. Tegmark, "Inflationary Constraints on Type IIA String Theory," JHEP 0712, 095 (2007) [arXiv:0711.2512 [hep-th]].

M. P. Hertzberg, M. Tegmark, S. Kachru, J. Shelton and O. Ozcan, "Searching for Inflation in Simple String Theory Models: An Astrophysical Perspective," Phys. Rev. D 76, 103521 (2007) arXiv:0709.0002 [astro-ph]].

[18] S. Hellerman, J. McGreevy and B. Williams, "Geometric constructions of nongeometric string theories," JHEP 0401, 024 (2004) arXiv:hep-th/0208174;

B. Wecht, "Lectures on Nongeometric Flux Compactifications," Class. Quant. Grav. 24, S773 (2007) arXiv:0708.3984 [hep-th]].

[19] A. Vilenkin, "Eternal inflation and chaotic terminology," arXiv:gr-qc/0409055.

[20] S. Kachru, R. Kallosh, A. Linde, J. M. Maldacena, L. McAllister and S. P. Trivedi, "Towards inflation in string theory," JCAP 0310, 013 (2003) arXiv:hep-th/0308055.

[21] D. Baumann, A. Dymarsky, I. R. Klebanov, L. McAllister and P. J. Steinhardt, "A Delicate Universe," arXiv:0705.3837 [hep-th].

[22] D. Baumann, A. Dymarsky, I. R. Klebanov and L. McAllister, "Towards an Explicit Model of D-brane Inflation," arXiv:0706.0360 [hep-th].

[23] S. Kachru, L. McAllister and R. Sundrum, "Sequestering in string theory," JHEP 0710, 013 (2007) arXiv:hep-th/0703105.

[24] S. B. Giddings, S. Kachru and J. Polchinski, "Hierarchies from fluxes in string compactifications," Phys. Rev. D 66, 106006 (2002) arXiv:hep-th/0105097.

[25] R. Kallosh and A. Linde, "Landscape, the scale of SUSY breaking, and inflation," JHEP 0412, 004 (2004) arXiv:hep-th/0411011. 
[26] V. Mukhanov, "Physical foundations of cosmology," Cambridge, UK: Univ. Pr. (2005) $421 p$

D. H. Lyth, "Particle physics models of inflation," Lect. Notes Phys. 738, 81 (2008) arXiv:hep-th/0702128.

[27] A. Linde and A. Westphal, "Accidental Inflation in String Theory," JCAP 0803, 005 (2008) [arXiv:0712.1610 [hep-th]].

[28] N. Iizuka and S. P. Trivedi, "An inflationary model in string theory," Phys. Rev. D 70, 043519 (2004) arXiv:hep-th/0403203.

[29] C. G. . Callan, C. Lovelace, C. R. Nappi and S. A. Yost, "String Loop Corrections To Beta Functions," Nucl. Phys. B 288, 525 (1987).

[30] P. Creminelli, L. Senatore, M. Zaldarriaga and M. Tegmark, "Limits on $f_{N} L$ parameters from WMAP 3yr data," JCAP 0703, 005 (2007) arXiv:astro-ph/0610600].

A. P. S. Yadav, E. Komatsu, B. D. Wandelt, M. Liguori, F. K. Hansen and S. Matarrese, "Fast Estimator of Primordial Non-Gaussianity from Temperature and Polarization Anisotropies in the Cosmic Microwave Background II: Partial Sky Coverage and Inhomogeneous Noise," arXiv:0711.4933 [astro-ph].

A. P. S. Yadav and B. D. Wandelt, arXiv:0712.1148 [astro-ph]. http://pirsa.org/speaker/Eiichiro_Komatsu http://pirsa.org/speaker/Kendrick_Smith

[31] X. Chen, M. x. Huang, S. Kachru and G. Shiu, "Observational signatures and non-Gaussianities of general single field inflation," JCAP 0701, 002 (2007) arXiv:hep-th/0605045.

C. Cheung, P. Creminelli, A. L. Fitzpatrick, J. Kaplan and L. Senatore, "The Effective Field Theory of Inflation," arXiv:0709.0293 [hep-th].

[32] R. Kallosh and A. Linde, "Testing String Theory with CMB," JCAP 0704, 017 (2007) arXiv:0704.0647 [hep-th]].

[33] D. Green, "Reheating Closed String Inflation," Phys. Rev. D 76, 103504 (2007) arXiv:0707.3832 [hep-th]].

[34] See e.g.

H. Jockers, "D-brane monodromies from a matrix-factorization perspective," JHEP 0702, 006 (2007) arXiv:hep-th/0612095;

P. S. Aspinwall and M. R. Douglas, "D-brane stability and monodromy," JHEP 0205, 031 (2002) arXiv:hep-th/0110071.

J. Distler, H. Jockers and H. j. Park, "D-brane monodromies, derived categories and boundary linear sigma models," arXiv:hep-th/0206242. 
[35] L. Kofman, A. Linde, X. Liu, A. Maloney, L. McAllister and E. Silverstein, "Beauty is attractive: Moduli trapping at enhanced symmetry points," JHEP 0405, 030 (2004) arXiv:hep-th/0403001.

[36] D. Green, B. Horn, T. Rube, L. Senatore, E. Silverstein: Work in progress;

N. Arkani-Hamed, private communication;

See also references on "Warm Inflation" such as

A. Berera and T. W. Kephart, "Ubiquitous inflaton in string-inspired models," Phys. Rev. Lett. 83, 1084 (1999) |arXiv:hep-ph/9904410];

I. G. Moss and C. Xiong, "Dissipation coefficients for supersymmetric inflatonary models," arXiv:hep-ph/0603266.

[37] N. Kaloper, M. Kleban, A. E. Lawrence and S. Shenker, "Signatures of short distance physics in the cosmic microwave background," Phys. Rev. D 66, 123510 (2002) arXiv:hep-th/0201158.

R. Easther, B. R. Greene, W. H. Kinney and G. Shiu, "Inflation as a probe of short distance physics," Phys. Rev. D 64, 103502 (2001) arXiv:hep-th/0104102].

[38] M. Alishahiha, E. Silverstein and D. Tong, "DBI in the sky," Phys. Rev. D 70, 123505 (2004) arXiv:hep-th/0404084.

X. Chen, "Multi-throat brane inflation," Phys. Rev. D 71, 063506 (2005) arXiv:hep-th/0408084].

R. Bean, X. Chen, H. V. Peiris and J. Xu, "Comparing Infrared Dirac-Born-Infeld Brane Inflation to Observations," Phys. Rev. D 77, 023527 (2008) arXiv:0710.1812 [hep-th]].

[39] See e.g.

T. Damour and A. Vilenkin, "Gravitational wave bursts from cusps and kinks on cosmic strings," Phys. Rev. D 64, 064008 (2001) arXiv:gr-qc/0104026].

N. T. Jones, H. Stoica and S. H. H. Tye, "The production, spectrum and evolution of cosmic strings in brane inflation," Phys. Lett. B 563, 6 (2003) [arXiv:hep-th/0303269].

E. J. Copeland, R. C. Myers and J. Polchinski, "Cosmic superstrings II," Comptes Rendus Physique 5, 1021 (2004).

M. G. Jackson, N. T. Jones and J. Polchinski, "Collisions of cosmic F- and D-strings," JHEP 0510, 013 (2005) arXiv:hep-th/0405229].

[40] B. Freivogel, M. Kleban, M. Rodriguez Martinez and L. Susskind, "Observational consequences of a landscape," JHEP 0603, 039 (2006) arXiv:hep-th/0505232]. 